\title{
Transcriptomic Analysis Reveals that Activating Transcription Factor 3/c-Jun/Lgals3 Axis Is Associated with Central Diabetes Insipidus after Hypothalamic Injury
}

\author{
Mingfeng Zhou ${ }^{a}$ Yichao $\mathrm{Ou}^{\mathrm{a}}$ Guangsen $\mathrm{Wu}^{\mathrm{a}}$ Kai $\mathrm{Li}^{\mathrm{a}}$ Junjie Peng ${ }^{\mathrm{a}}$ \\ Xingqin Wang ${ }^{a}$ Mengjie Che ${ }^{a}$ Haodong Gong ${ }^{b}$ Peirong Niub Yawei Liu ${ }^{a}$ \\ Zhanpeng Feng ${ }^{\mathrm{a}}$ Songtao $\mathrm{Qi}^{\mathrm{a}}$ \\ aDepartment of Neurosurgery, Nanfang Hospital, Southern Medical University, Guangzhou, PR China; ${ }^{\text {b The First }}$ \\ School of Clinical Medicine, Southern Medical University, Guangzhou, PR China
}

\section{Keywords}

Hypothalamic injury · Central diabetes insipidus ·

Transcriptome

\begin{abstract}
Background: Hypothalamic injury causes several complicated neuroendocrine-associated disorders, such as waterelectrolyte imbalance, obesity, and hypopituitarism. Among these, central diabetes insipidus (CDI), characterized by polyuria, polydipsia, low urine specific gravity, and deficiency of arginine vasopressin contents, is a typical complication after hypothalamic injury. Methods: CDI was induced by hypothalamic pituitary stalk injury in male animals. Behavioral parameters and blood sample were collected to evaluate the characteristics of body fluid metabolism imbalance. The brains were harvested for high-throughput RNA sequencing and immunostaining to identify pathophysiological changes in corresponding hypothalamic nuclei. Results: Based on transcriptomic analysis, we demonstrated the upregulation of the activating transcription factor 3 (Atf3)/c-Jun axis and identified Lgals3, a microglial activation-related gene, as the most significant target gene in response to the body fluid imbalance in CDI. Furthermore, we found that the microglia
\end{abstract}

karger@karger.com www.karger.com/nen

Karger $\stackrel{\text { ' }}{=}$

BOPEN ACCESS
(C) 2021 The Author(s)

Published by S. Karger AG, Basel

This is an Open Access article licensed under the Creative Commons Attribution-NonCommercial-4.0 International License (CC BY-NC) (http://www.karger.com/Services/OpenAccessLicense), applicable to the online version of the article only. Usage and distribution for commercial purposes requires written permission. possessed elevated phagocytic ability, which could promote the elimination of arginine vasopressin neurons after hypothalamic injury. Conclusion: Our findings suggested that the Atf3/c-Jun/Lgals3 axis was associated with the microglial activation, and might participate in the loss of functional arginine vasopressin neurons in CDI after hypothalamic injury.

(c) 2021 The Author(s).

Published by S. Karger AG, Basel

\section{Introduction}

The hypothalamus weighs only $4 \mathrm{~g}$, accounting for approximately $0.3 \%$ of the entire brain, yet it serves as a primary structure of the autonomic nervous system, limbic system, reticular activating system, and hypothalamuspituitary endocrine in human. As a cross-regulation center of the nervous and endocrine systems, the hypothalamus governs various physiological processes including hydromineral balance, light-dark cycle, autonomic tonus, sexual behavior, and reproduction $[1,2]$. Hydromineral

Mingfeng Zhou, Yichao Ou and Guangsen Wu have contributed equally to this work.
Correspondence to:

Zhanpeng Feng, feng3388836@smu.edu.cn

Songtao Qi, qisongtaonfyy@126.com 
regulatory peptidergic neuronal cell bodies are concentrated in several hypothalamic nuclei including supraoptic nucleus (SON) and paraventricular nucleus (PVN), which could produce and secrete their products into the circulation, such as arginine vasopressin (AVP) and oxytocin (OXT), through the neurohypophysis. The main peripheral target of AVP is located in the thick ascending limb of Henle's loop and the collecting duct in the renal system, and AVP could promote the expression of water channel, thus increasing water permeability and reabsorption. OXT binds to OXT receptor in myometrium, stimulates the release of atrial natriuretic peptide from the heart, and finally produces a diuretic response through modulation of sodium channels in distal nephron cells [3]. The hypothalamus also secretes other neuronal hormones including gonadotropin-releasing hormone, and prolactin, thus participating in gonad development, sexual activity, pregnancy, delivery, and lactation processes $[4,5]$.

Neural injury caused by trauma, radiation, and surgical disruption can trigger a cascade of cellular events, resulting in extensive damage and ultimately neuronal apoptosis, axonal Wallerian degeneration, and glial reaction in related diseases, including Parkinson's disease, Alzheimer's disease, Huntington's disease, and traumatic brain injury, especially axonal injury [6, 7]. Hypothalamic injury, a devastating type of central nervous system (CNS) injury, can not only cause acute hypothalamic reaction, including extremely high fever and circulation disorders, but can also lead to serious neuroendocrine impairments, such as water-electrolyte imbalance and obesity, because of its vital role in neural-endocrine regulation [8-11]. Central diabetes insipidus (CDI) is a waterelectrolyte disorder, characterized by polyuria, polydipsia, low urine specific gravity (USG), and decrease of AVP contents. CDI serves as a common complication after hypothalamic injury, especially pituitary stalk injury during resection of tumors near the hypothalamus, such as craniopharyngioma and pituitary tumor [12-14]. As a consequence of pituitary stalk injury, loss of magnocellular AVP and OXT neurons is a key feature of hypothalamic injury-induced CDI. However, the detailed hydromineral imbalance pattern and underlying mechanism are not fully understood.

Various studies have addressed gene expression changes in CNS trauma models, including spinal cord injury and optic nerve crush [15-17], and identified the involvement of several processes, such as inflammation, immune regulation, axonal degeneration, and apoptosis [18]. In terms of hypothalamic regulation of hydromin- eral metabolism, researchers have demonstrated global gene expression changes of the hypothalamic-neurohypophyseal system in response to hyperosmotic and hypoosmotic stimuli and provided insights into the quantitative assessment and screening of regulatory genes in specific hypothalamic tissue [19-22]. However, there have been no such studies evaluating global gene expression patterns and verifying the key associated mechanism pathways in hypothalamic injury-induced hydromineral imbalance including CDI.

In this study, we comprehensively investigated gene expression changes, determined the related transcription factor network, and identified several important downstream biological processes including magnocellular neuronal loss and underlying mechanism in CDI, using a hypothalamic-pituitary stalk lesion model.

\section{Materials and Methods}

\section{Animals}

All male 8-10-week-old C57BL/6J mice and Sprague-Dawley rats with an average body weight of $200 \mathrm{~g}(180-220 \mathrm{~g})$ were housed in independent metabolic cages (DXL-XS, FENGSHI, SuZhou, China) with daily light and dark cycle in a temperature-controlled room. During the whole experiment, food and water were provided ad libitum. Animals were allocated randomly to each experimental group. Animals which died after surgery were excluded for further analysis. Rats were used for RNA-sequencing and Western blotting considering the precise acquisition of hypothalamic nuclei and enough contents of extracted RNA and protein, while the rest experiments were mainly performed in mice.

\section{Hypothalamic Injury Model Construction: Pituitary Stalk}

\section{Electric Lesion}

All animals were allowed to acclimatize to their environment in independent metabolic cages for 3 days before surgery. Pituitary stalk electric lesion (PEL) surgery for mice was performed based on previous studies [23-25]. In brief, mouse was deeply anesthetized with $75 \mathrm{mg} / \mathrm{kg}$ sodium pentobarbital. After shaving and disinfection, mouse head was mounted on a stereotaxic frame (RWD Life Science Inc., Shenzhen, China). Then, the skull was slightly adjusted to keep the bregma and lambda horizontally and opened by removing a $1 \times 1 \mathrm{~mm}^{2}$ (approx.) of bone at 1.9 - $\mathrm{mm}$ caudal to bregma in the sagittal midline. Next, a 3D-printed lesion knife, with a 0.8 -mm-wide curved head and 0.5 -mm-thickness, was lowered in the sagittal midline until it reached the floor of the skull base which was over $6 \mathrm{~mm}$ beneath the surface of the skull. After compression for $60 \mathrm{~s}$, a cathodic current of $0.3 \mathrm{~mA}$ was applied for $30 \mathrm{~s}$ with a constant power supply output (53500; UGO Basile, Gemonio, Italy), while the injury depth was $5 \mathrm{~mm}$ and no current was applied in control group. After surgery, all mice were put back to the original metabolic cages and their daily water consumption (DWC), daily urine volume (DUV), and USG were monitored for 28 days. PEL surgery for rats was performed similarly according to our previous study [23]. 


\section{Index Calculation}

First, using k-means cluster analysis, the daily status of the mouse was classified into diabetes insipidus condition and nondiabetes insipidus condition defined as the control mice, based on the DWC, DUV, and USG. Then, the CDI index was defined as the portion of the days that are clustered into diabetes insipidus condition to the total days during a specific period. The clustered results between PEL and control group were further visualized using $\mathrm{R}$ package: prcomp, ggplot2 [26].

\section{Cannula Implantation and Drug Delivery}

For intracerebral ventricle delivery of drug, mice received a guide cannula fixed into the right lateral ventricle with 1 small screw and dental acrylic after PEL surgery, using the following stereotaxic coordinates: $-0.5 \mathrm{~mm}$ anterior-posterior, $+1.1 \mathrm{~mm}$ medial-lateral, $-2.5 \mathrm{~mm}$ below the surface of the skull according to the Mouse Brain Stereotaxic Atlas [27]. A stainless-steel probe with a plastic cap was inserted into each cannula to keep them blocked and prevent infection. During the injection period, a stainless injection cannula was inserted into the guide cannula and $5 \mu \mathrm{L}$ inhibitor of activating transcription factor 3 (Atf3)/c-Jun, SP600125 (1 $\mu \mathrm{g} / \mu \mathrm{L}$, Selleck, China) was administered using a microinfusing pump at the rate of $2 \mu \mathrm{L} / \mathrm{min}$ once a day for 3 or 7 continuous days after PEL surgery according to the experimental design. The injection cannula was left in place for 5 additional minutes to allow the diffusion of drug and prevent the backflow through the cannula tract. Cannula localization was confirmed by injection of dye followed by acute slicing. Only brains with clear cannula track into lateral ventricular were used for further analysis.

\section{Perfusion and Tissue Sectioning}

At the end of experiment, mice were deeply anesthetized with sodium pentobarbital $(75 \mathrm{mg} / \mathrm{kg})$, and perfused intracardially with ice-cold normal saline, followed by cool $4 \%$ paraformaldehyde in $1 \mathrm{X}$ phosphate buffer solution (PBS) ( $\mathrm{pH} 7.4)$. After removed from the skull, brains were placed in $4 \%$ formaldehyde overnight followed by sequential $15 \%$ and $30 \%$ sucrose at $4^{\circ} \mathrm{C}$ for at least $24 \mathrm{~h}$, respectively. Then, brains were embedded in O.C.T. compound, and coronal sections were cut at $30 \mu \mathrm{m}$ using freezing microtome (Leica, 1850S). All sections were collected and preserved in PBS containing $0.05 \%$ sodium azide at $4^{\circ} \mathrm{C}$ for further immunostaining.

\section{Immunofluorescence}

Coronal brain sections were rinsed with PBS, blocked with 5\% nonspecific antigen goat serum containing $0.5 \%$ Triton X-100 for $1 \mathrm{~h}$ at $37^{\circ} \mathrm{C}$, and incubated overnight at $4^{\circ} \mathrm{C}$ with a specific primary antibody diluted in $5 \%$ goat serum containing $0.2 \%$ Triton $\mathrm{X}-100$. The next day, after rinsed with $\mathrm{PBS}$ containing $0.2 \%$ Triton $\mathrm{X}-100$ for 3 times, sections were incubated for $1 \mathrm{~h}$ at $37^{\circ} \mathrm{C}$ with corresponding secondary antibodies conjugated with Alexa-488 or Alexa-594 (Thermo Fisher Scientific, Waltham, MA, USA) diluted in $5 \%$ goat serum containing $0.2 \%$ Triton X-100. After staining with 4-6-diamidino-2-phenylindole, all sections were slowly mounted on glass slides and cover glasses were slipped in mounting medium. Fluorescent images were captured with confocal microscope (LSM980; Zeiss, Oberkochen, Germany) using ZEN software (Blue edition, version 3.2). Labeled cells were counted using ImageJ software (NIH). For specific cell number counting, data were presented as the number of cells/slice. For double staining, data were presented as the ratio of double-positive cells to the single-positive cells.

\section{Immunolabeling-Enabled 3D Imaging of Solvent-Cleared} Organs

For immunolabeling-enabled 3D imaging of solvent-cleared organs (iDISCO) imaging, mice were perfused and brains were collected as above mentioned. Then, the whole hypothalamus tissue samples (ranging from bregma $+0.50 \mathrm{~mm}$ to $-1.50 \mathrm{~mm}$ ) were acquired using a razor blade and treated as previous studies [28, 29]. Briefly, mouse hypothalamus samples were dehydrated by gradient methanol, consisting of $20 \%, 40 \%, 60 \%, 80 \%, 100 \%$ methanol in distilled water, $1 \mathrm{~h}$ for each step. Next, samples were bleached by $5 \%$ hydrogen peroxide in methanol at $4^{\circ} \mathrm{C}$ overnight. Following rehydrated in gradient decrease methanol, samples were permeabilized by $20 \%$ dimethylsulfoxide in PBS for 1 day and blocked with goat serum for another day at $37^{\circ} \mathrm{C}$. Then, samples were incubated with primary antibodies (rabbit anti-Iba1) and corresponding secondary antibodies (goat anti-rabbit Alexa Fluor 594-conjugated) for 3 days at $37^{\circ} \mathrm{C}$, respectively. After washed with PBS, immunolabeled samples were cleared with dicholoromethane (Sigma) for $3 \mathrm{~h}$, immersed in dibenzyl ether (Sigma) until clear, and stored in dibenzyl ether for further imaging. All images for tissue volume were acquired with multiphoton microscope (FV1200MPE; Olympus, Tokyo, Japan) and 3D simulation was generated using Imaris software (Bitplane, version 8.5).

\section{Western Blotting}

PEL-treated rats were perfused with ice-cold normal saline, and hypothalamus tissues including SON and PVN were collected under a stereomicroscope. Hypothalamic tissues were washed thoroughly with PBS, lysed with RIPA buffer (50 mM Tris- $\mathrm{HCl} \mathrm{pH} \mathrm{8.0,}$ $1 \mathrm{mM}$ EDTA pH 8.0, $5 \mathrm{~mm}$ DTT, 2\% SDS) containing protease inhibitor and phosphoric-acid protease inhibitor using low temperature tissue homogenizer, and kept under $4^{\circ} \mathrm{C}$ for $30 \mathrm{~min}$. The protein concentration was determined by BCA assay (Beyotime Inc, Xiamen, China). Protein samples were subsequently separated using SDS-PAGE gel and electro-transferred to polyvinylidene difluoride membranes (Millipore, Burlington, MA, USA). After blocked with $5 \% \mathrm{BSA}$, the membranes were incubated with specific primary antibodies overnight at $4^{\circ} \mathrm{C}$, and then incubated with corresponding horseradish peroxidase conjugated secondary antibody for $1 \mathrm{~h}$ under room temperature. Finally, signal was detected using enhanced chemiluminescence reagents (Millipore, USA) and captured by a digital camera.

\section{Real-Time Quantitative PCR}

For real-time quantitative PCR, total RNA was extracted from the freshly frozen mice hypothalamic nuclei acquired under stereomicroscope using TRIzol reagent (Takara, Beijing, China). RNA concentration and purity were checked using NanoPhotometer 2000. Only qualified RNA samples (OD260/280 ranging from 1.9 to 2.1 to avoid genomic DNA contamination) were reverse transcribed with a cDNA synthesis kit (Takara, RR047A), which contains genomic DNA eraser regents. Real-time quantitative PCR was further performed with a corresponding amplification kit (Takara, RR820A), based on a 2-step method. The optimization and validation of primers were performed using a real-time quantitative PCR standard curve with 5 concentrations and a dilution factor of 1:3. 
The primers' efficiencies vary between $103.9 \%$ and $112.9 \%$. In accordance with previous studies showing the stable expression of ribosomal protein L19 mRNA (Rpl19) after osmotic stimulus [19], the average cycle thresholds for Rpl19 in this study were $21.80 \pm$ $0.07,20.87 \pm 0.05,20.69 \pm 0.12$, and $20.75 \pm 0.12$ for the control, PEL_3d, PEL_7d, and PEL_28d groups, respectively. Thus, Rpl19 was used as internal control gene for relative quantification of specific gene expression and the fold changes were analyzed using comparative $\left(2^{-\Delta \Delta \mathrm{Ct}}\right)$ method. The following related primers were used TNF- $\alpha$ forward: 5' - -3' GGTGCCTATGTCTCAGCCTCTT, TNF- $\alpha$ reverse: $5^{\prime}-$ - $^{\prime}$ GCCATAGAACTGATGAGAGGGAG; IL$1 \beta$ forward: $5^{\prime}-3^{\prime}$ TGGACCTTCCAGGATGAGGACA, $I L-1 \beta$ reverse: $5^{\prime}-3^{\prime}$ GTTCATCTCGGAGCCTGTAGTG; $i N O S$ forward: $5^{\prime}-$ - $^{\prime}$ GAGACAGGGAAGTCTGAAGCAC, iNOS reverse: $5^{\prime}--3^{\prime}$ CCAGCAGTAGTTGCTCCTCTTC; Arg1 forward: $5^{\prime}-\quad-3^{\prime}$ CATTGGCTTGCGAGACGTAGAC, Arg1 reverse: 5' - -3' GCTGAAGGTCTCTTCCATCACC; Rpl19 forward: $5^{\prime}--3^{\prime}$ GCGTCTGCAGCCATGAGTA, Rpl19 reverse: $5^{\prime}-$ - $3^{\prime}$ TGGCATTGGCGATTTCGTTG.

\section{Antibodies}

The following primary antibodies were used: rabbit anti-AVP (AB1565; Millipore), mouse anti-AVP (MABN845, Millipore); rabbit anti-Atf3 (A13469; Abclonal, Woburn, MA, USA); rabbit anti-p-c-Jun (3270; Cell Signaling Technology, Danvers, MA, USA); mouse anti-p-c-Jun (sc-822; Santa Cruz, Dallas, TX, USA); mouse anti-Lgals3 (60207-1; Proteintech, Rosemont, IL, USA); and rabbit anti-Iba1 (ab153696; Abcam, Cambridge, UK).

\section{MRI Scanning}

For magnetic resonance imaging (MRI) scanning, mice were anaesthetized with isoflurane, and scanning was performed using 7.0 T Bruker Pharmascan (Bruker Biospin, Billerica, MA, USA) equipped with a 6-cm volume resonator using Paravision 5.0. T1weighted images were acquired after intraperitoneal injection of $50 \mu \mathrm{L} \mathrm{Gd}$-DTPA. The following parameters were used: $\mathrm{TR}=3,000$ $\mathrm{ms}, \mathrm{TE}=36.0 \mathrm{~ms}$ for T2 images; $\mathrm{TR}=1,500 \mathrm{~ms}, \mathrm{TE}=8.0 \mathrm{~ms}$ for $\mathrm{T} 1$ images. Slice thickness $=0.5 \mathrm{~mm}$.

\section{Serum AVP Content Measurements}

At different time after PEL surgery, C57BL/6J male mice were deeply anesthetized with $75 \mathrm{mg} / \mathrm{kg}$ sodium pentobarbital. Blood samples were collected from the right ventricle and separated by centrifugation at $1,500 \mathrm{rpm}$ for $10 \mathrm{~min}$ for serum. The AVP contents were detected following the manufacturer's instructions by using ELISA kit (74-VSPHU-E01.1, ALPCO).

\section{RNA Preparation for High-Throughput RNA-Sequencing}

For accurate acquisition and adequate RNA content extraction for sequencing, hypothalamic tissues containing SON and PVN for high-throughput RNA-sequencing were harvested in rat after PEL surgery based on our previous study [25]. Briefly, rats were sacrificed at 1 day or 7 day after PEL surgery, and hypothalamic tissues were dissected in ice-cold PBS. SON and PVN were acquired using forceps under stereomicroscope. Hypothalamic SON and PVN tissues were pooled together and homogenized with TRIzol reagent to extract total RNA according to the reagent's manufacturer instruction. RNA purity was checked using NanoPhotometer R spectrophotometer and RNA concentration was measured using Qubit R RNA Assay Kit in Qubit R 2.0 Flurometer.
Finally, RNA integrity was assessed using the RNA Nano 6000 Assay Kit of the Bioanalyzer 2100 system (Agilent Technologies, Santa Clara, CA, USA).

\section{Library Preparation and Sequencing}

RNA samples from 3 biological replicates were purified using poly-T oligo-attached magnetic beads. Then, sequencing libraries were conducted using NEBNext R UltraTM RNA Library Prep Kit for Illumina $\mathrm{R}$ (NEB) according to the manufacturer's instructions. The library fragments were purified with AMPure XP system (Beckman Coulter, Brea, CA, USA) for cDNA fragments of preferentially $250-300 \mathrm{bp}$ in length. The library preparations were sequenced on an Illumina Hiseq platform, and $125 \mathrm{bp} / 150 \mathrm{bp}$ paired-end reads were generated with quality assessed.

\section{Gene Expression Analysis}

The RNA-seq reads were aligned to the reference genome using Hisat2 v2.0.5. Feature Counts v1.5.0-p3 was used to count the read numbers mapped to each gene. Differential expression analysis was performed using the DESeq2 method (version 1.10.1). The resulting $p$ value was adjusted using the Benjamini and Hochberg's approach for controlling the false discovery rate $(<0.05)$. Genes with an adjusted $p$ value $<0.05$ (detected by DESeq2) between different groups were considered to be differentially expressed.

\section{Functional Enrichment Analysis of Differentially Expressed} Genes

The differentially expressed genes (DEGs) between different groups (PEL_day 1 vs. control, PEL_day 7 vs. control) with adjusted $p$ value $<0.05$ were further analyzed using the publicly available bioinformatics software Database for Annotation, Visualization and Integrated Discovery. Several gene ontology (GO) terms were identified based on cluster analysis with Benjamini multiple test correction $p<0.05$. The gene set enrichment analysis (GSEA) and $\mathrm{GO}$ analysis results were further visualized by R package: clusterProfiler [30].

\section{Differentially Expressed TFs Identification and Prediction of}

Target Genes

Transcription factor (TF) genes were acquired from online database AnimalTFDB v3.0 (http://bioinfo.life.hust.edu.cn/AnimalTFDB/\#!/). DEGs between different groups were compared with the TF list, and common genes were identified as differentially expressed TFs. Target genes of interested TF were predicted by an online web server (http://cistrome.org/db/\#/), a common database for putative target gene searching. Putative target genes were superposed with the DEGs to gain an intersection for further analysis.

\section{Protein-Protein Interaction Network and Core Module} Construction

The common DEGs between different groups, and b-ZIP family TFs were analyzed using the online database STRING (https:// string-db.org/) for protein-protein interaction (PPI) network construction. Co-expression network was analyzed by molecular complex detection, a plugin in Cytoscape that clustered a given network based on topology to find densely connected regions, and the most significant cluster with 10 nodes. 

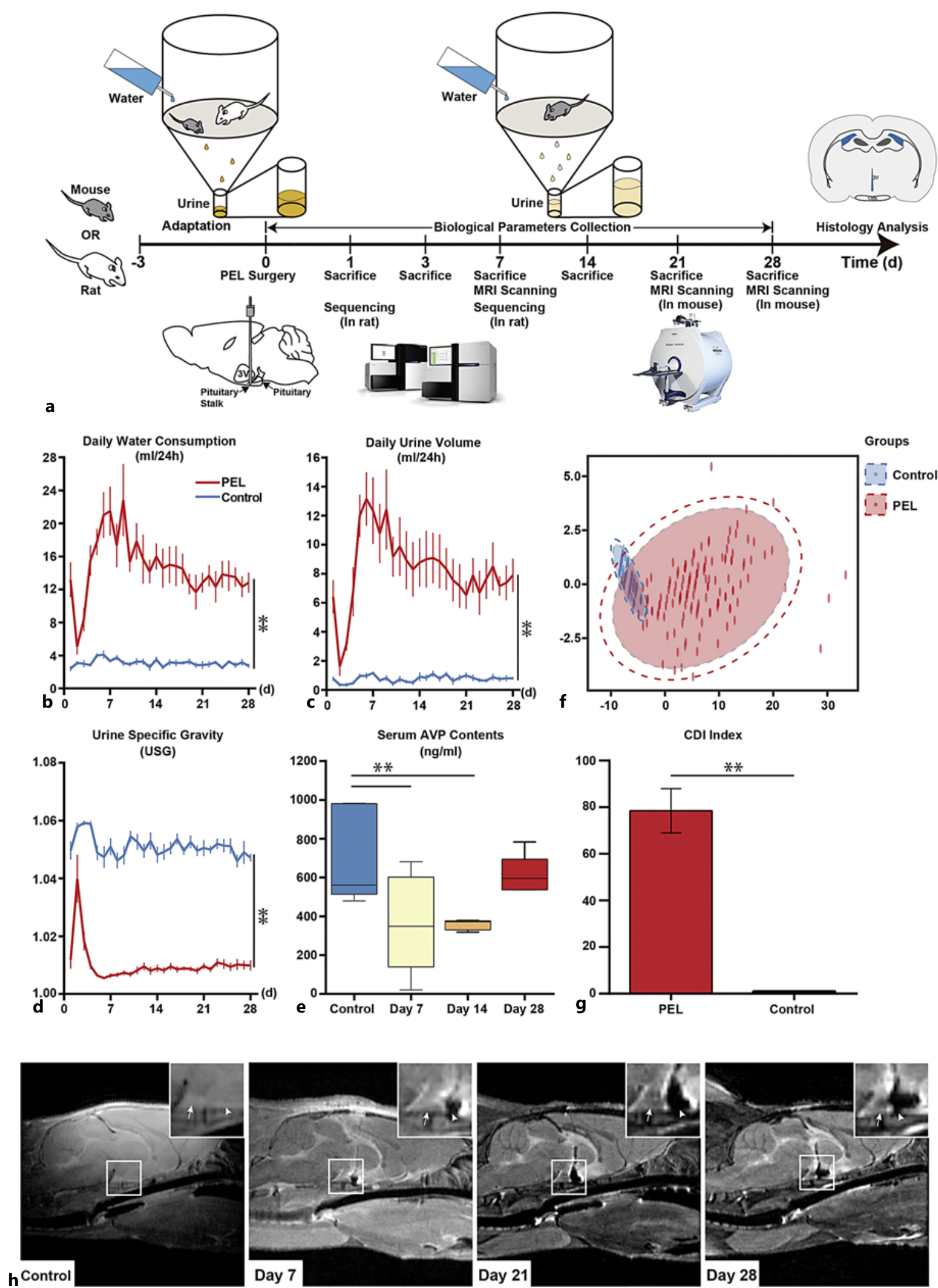

1

(For legend see next page.) 
Statistical Analysis

Data were submitted to Kolmogorov-Smirnov and ShapiroWilk normality test to identify the distribution. Two-tailed Student's $t$ test and Mann-Whitney U test were used for single comparison between 2 groups. The rest of the data were analyzed by 1-way ANOVA followed by LSD correction and Kruskal-Wallis test followed by Dunn's multiple comparisons. All statistical analyses were performed using SPSS software (version 23.0, IBM, Armonk, NY, USA). Error bars in all figures represent the mean \pm SEM. $p<0.05$ was considered significant.

\section{Results}

\section{CDIs after Hypothalamic-Pituitary Stalk Injury}

To carry out intensive study into hypothalamic injuryinduced hydromineral imbalance, we constructed a hypothalamic-PEL model using a transparietal approach that did not interfere with the anterior pituitary lobe and hypophyseal portal system using a 3-dimensional (3D)printed knife combined with an electrolytic lesion. As shown in Figure 1a, we performed PEL surgery on day 0 and MRI in mouse at serial time points thereafter to visualize the hypothalamic structure. In addition, we also performed RNA-seq analysis of the hypothalamic nuclei in PEL-treated rats to identify potentially related genes expression pattern. During the entire experiment, 3 waterelectrolyte metabolism-related biological parameters, DWC, DUV, and USG, were monitored to evaluate daily status. Compared with control mice, PEL-treated mice exhibited a typical tri-phasic CDI pattern. The first day was classified as the acute phase of CDI with high DWC and DUV and low USG. DWC and DUV dropped dramatically and gradually increased from post-surgery day 2 to day 7 , which was identified as the second phase of CDI. The third phase of CDI was defined from day 7, with persistently high DWC $(t=8.081$; d.f. $=10$; $p<0.01$; Fig. 1b) and DUV ( $t=6.146$; d.f. $=10 ; p<0.01$; Fig. 1c) and low USG $(t=16.942$; d.f. $=10 ; p<0.01$; Fig. $1 \mathrm{~d})$ for an extended period. Serum AVP levels also showed a rapid decrease in the acute phase, followed by gradual recov-

Fig. 1. Typical CDI phenotype induced by hypothalamic PEL. The whole experimental scheme of this study (a), DWC (b), DUV (c), and USG $(\mathbf{d})$ in PEL-treated mice and control mice $(n=6)$. Serum AVP contents on days 7, 21, and 28 were detected by ELISA $(n=$ 5) (e). Daily status was classified into diabetes insipidus or normal condition based on the DWC, DUV, and USG using k-means cluster method and visualized by PCA plot (f). CDI index was defined as the ratio of diabetes insipidus condition to the total days $(\mathbf{g})$. MRI scanning images showed the transection site of pituitary stalk

Transcriptomic Analysis of Central

Diabetes Insipidus ery $\left(F_{|3,16|}=4.592 ; p<0.05\right.$; Fig. 1e), indicating the disruption of AVP synthesis, transportation, or secretion into circulation after hypothalamic pituitary stalk injury. Next, using the k-means cluster method, we defined the CDI index as the ratio of daily status classified into diabetes insipidus condition to the whole experimental period based on the DWC, DUV, and USG. Compared with control mice, PEL-treated mice showed a distinct pattern with a CDI index near $80 \%(H=3.102$; $p<0.01$; Fig. 1f-g). Additionally, MRI also confirmed the complete interruption of the pituitary stalk (arrowhead) and weakened signal of the pituitary showed in T1-weighted images after PEL surgery (Fig. 1h).

\section{AVP Neuronal Loss after Hypothalamic Injury}

To investigate the underlying mechanism of hypothalamic injury, we extracted RNA for high-throughput sequencing from rat hypothalamic SON and PVN, which concentrate several hydromineral regulatory peptidergic neurons on day 1 and day 7 after PEL surgery. Original RNA seq data can be acquired from the pubic GEO database (GSE167904). Based on RNA-seq analysis, we identified 1,711 DEGs (1,292 upregulated and 419 downregulated) between the PEL_1d (1 day after PEL) and control groups, and 185 DEGs (150 upregulated and 35 downregulated) between the PEL_7d (7 day after PEL) and control groups (defined as adjusted $p$ values $<0.05$ ). Among the top 20 upregulated DEGs, we identified several overlapping injury-associated genes, such as Atf3, Sprr1a, Triap1, and the well-known microglial activation-related gene, Lgals3. Furthermore, Avp was the most downregulated gene in the PEL_7d group compared with the control group, indicating the abnormality of AVP expression and synthesis in the hypothalamo-neurohypophyseal system (Fig. 2a). Therefore, we further performed AVP staining in the mouse CDI model to validate the pathophysiological changes in the hypothalamic SON and PVN. As shown in Figure 2b, AVP neuron counts showed a progressive decrease to 35.2/slice on day 3 and 21.3/slice on day 7 in SON after PEL surgery compared

(arrowhead) and weaken signal in posterior lobe of pituitary (arrow) in mouse hypothalamus after PEL surgery (h). Data are analyzed by unpaired $t$ test (b-d), Mann-Whitney U test (g) and 1-way ANOVA followed by LSD multiple comparisons test (e). Data are presented as mean \pm SEM. ${ }^{* *} p<0.01$ compared with control group. PEL, pituitary stalk electrical lesion; DWC, daily water consumption; DUV, daily urine volume; USG, urine specific gravity; CDI, central diabetes insipidus; AVP, arginine vasopressin; MRI, magnetic resonance imaging. 

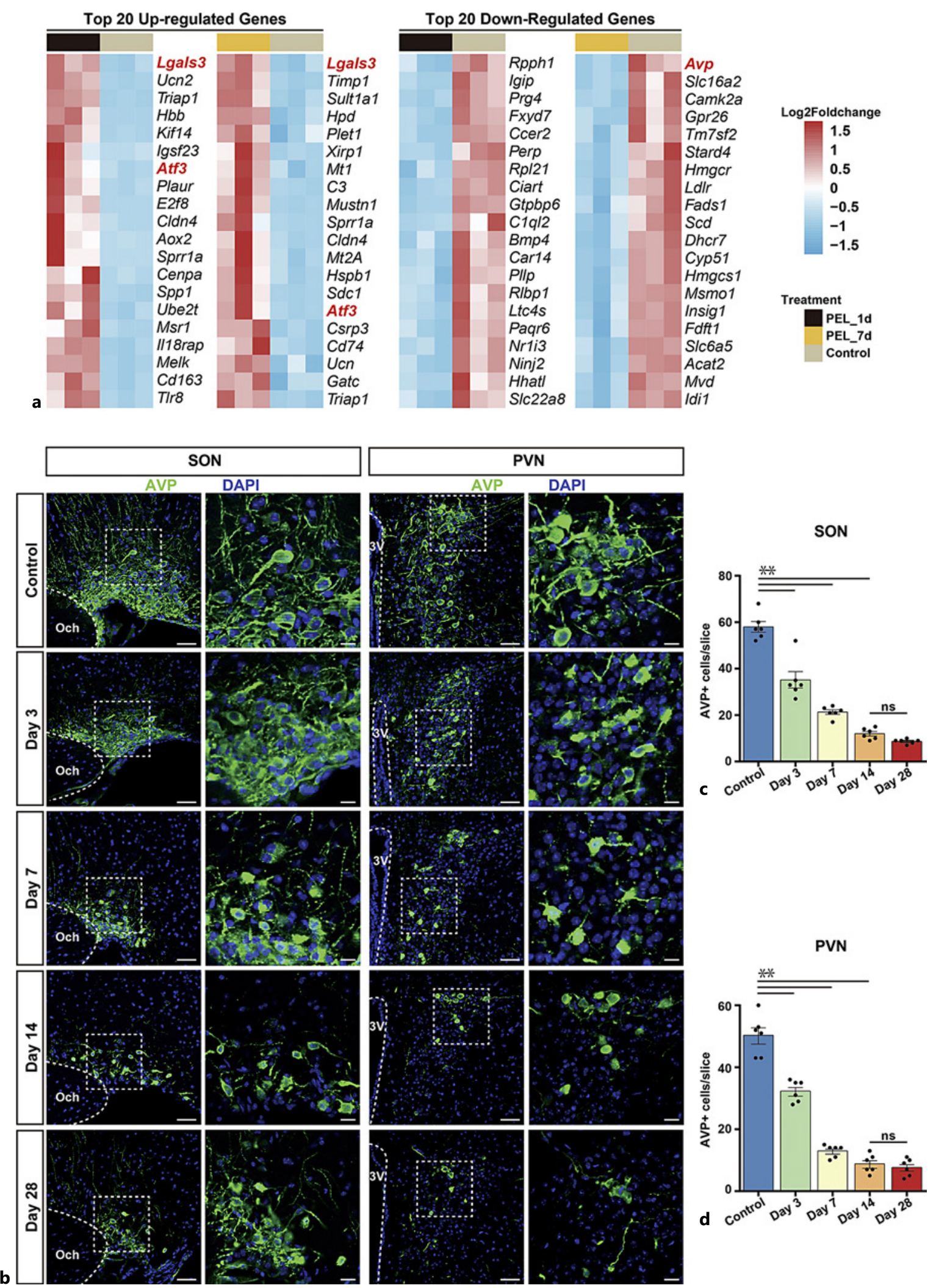

2

(For legend see next page.) 
with 58.0/slice in the control group $\left(F_{|4,25|}=101.731 ; p<\right.$ 0.01; Fig. 2c). AVP neurons in the PVN also showed a significant decrease from 50.3/slice in the control group to 32.3 /slice on day $3,13.0$ /slice on day 7 , and 8.8 /slice on day 14 after PEL surgery $\left(F_{|4,25|}=136.903 ; p<0.01\right.$; Fig. 2d). To fully assess the hypothalamo-neurohypophyseal system, we further performed sagittal AVP axon staining from the hypothalamus to the pituitary. Consistently, compared with strong AVP staining in the control group, almost no AVP-positive neuronal fibers were found along the floor of third ventricular near the hypothalamus, and median eminence, nor were AVP-positive storage granules present in neurohypophysis after PEL surgery (online suppl. Fig. S1; for all online suppl. material, see www.karger.com/doi/10.1159/000520865).

\section{Upregulation of the Atf3/c-Jun/Lgals3 Axis Based on RNA-Seq Analysis}

To identify potential mechanisms underlying AVP neuronal loss in CDI, we analyzed the DEGs from RNAseq data using the public online data-mining tool, Database for Annotation, Visualization and Integrated Discovery [31], to cluster all DEGs from the different groups into $3 \mathrm{GO}$ categories, i.e., biological process (BP), molecular function (MF), and cellular component. Enriched terms were further visualized using $\mathrm{R}$ software. Several transcription-related processes, including positive regulation of transcription from RNA polymerase II promoter (GO: 0045944), positive regulation of transcription, DNA-templated (GO: 0045893), translation repressor activity, and nucleic acid binding (GO: 0000900), ranked the top 10 clustered terms in the $\mathrm{BP}$ and MF categories in the PEL_1d group. Other enriched GO terms included positive regulation of cell proliferation (GO: 0008284) and response to wound (GO: 0009611) (Fig. 3a-b). Consistently, GSEA also showed that proliferation-associated processes, such as mitotic metaphase plate progression and regulation of chromosome segregation and cell cycle, were upregulated after PEL surgery (Fig. 3c-d). GO analysis between the PEL_7d and control groups further revealed the enrichment of neural repair-related terms, such as positive regulation of cell proliferation and sterol

Fig. 2. RNA-seq analysis reveals the hypothalamic AVP neuronal loss. Heatmaps of the top 20 significantly DEGs between PEL_1d, PEL_7d and control groups in rat CDI model $(n=3$, adjusted $p$ value $[p$-adj] $<0.05)(\mathbf{a})$. Validation of AVP neuronal loss in mouse hypothalamic SON and PVN after PEL surgery (b). Quantification results of residual AVP neurons in SON (c) $(n=6)$. Quantification results of residual AVP neurons in PVN $(\mathbf{d})(n=6)$. Scale bars, 50 biosynthetic process. Additionally, chemokine and cytokine signaling pathways were also upregulated based on GSEA analysis (Fig. 3e-f). The enriched transcriptionrelated processes indicate that transcription factor might be involved in the cell proliferation activation, acute phase wound response, and tissue remodeling in CDI after hypothalamic injury.

Next, we analyzed the intersection between the transcription factor downloaded from the online database AnimalTFDB v3.0 and DEGs from our RNA-seq data [32]. Surprisingly, we found that Atf 3 was the only transcription factor significantly upregulated both in the PEL_1d and PEL_7d groups compared with the control group (Fig. 4a). Co-staining experiments further demonstrated the elevated expression of Atf3 in AVP-producing neurons (Fig. 4b), with double-positive ratios of $70.7 \%$ on day $1,51.4 \%$ on day $3,17.9 \%$ on day 7 , and $7.7 \%$ on day 14 after PEL surgery in the mouse hypothalamic SON $\left(F_{\mid 4}\right.$, ${ }_{25}=150.599 ; p<0.01$; Fig. 4c). The same phenomenon was also found in the PVN, while nearly no Atf3 expression was found in the control group $(H=21.333,4 \mathrm{df}$; $p<0.01$; Fig. 4 d).

As an injury-responsive transcription factor, Atf3 belongs to the canonical b-ZIP transcription factor family and regulates the expression of its target genes by binding other b-ZIP family transcription factors. Based on the target genes obtained from the online Cistrome database, we found that 9 DEGs were potential target genes of Atf3 [33]. Among these, Lgals3 was identified as the most upregulated gene with a nearly 9.8-fold change in PEL_1d and a 6.6-fold change at PEL_7d compared with the control group (Fig. 4e). A PPI network constructed from 9 putative Atf3 target genes and other b-ZIP family transcription factors revealed the regulatory network of Atf 3 / Jun/Lgals3 (Fig. 4f). Moreover, the core cluster of the PPI network detected by molecular complex detection consisted of 10 genes and was visualized using the Cytoscape software. Among the clustered core modules, Atf3/Jun/ Lgals 3 was also identified as the most significant regulatory pathway (Fig. 4g). Co-localization of Atf3 and p-cJun (activated form of c-Jun encoded by Jun) and a similar upregulated expression pattern of $\mathrm{p}$-c-Jun in mouse $\mu \mathrm{m}$ for low magnification images and $20 \mu \mathrm{m}$ for high magnification images. Data are analyzed by 1-way ANOVA followed by LSD multiple comparisons test $(\mathbf{c}, \mathbf{d})$. Data are presented as mean \pm SEM. ${ }^{* *} p<0.01$ compared with control group. CDI, central diabetes insipidus; AVP, arginine vasopressin; DEGs, differentially expressed genes; SON, supraoptic nucleus; PVN, paraventricular nucleus; DAPI, diamidino-2-phenylindole. 

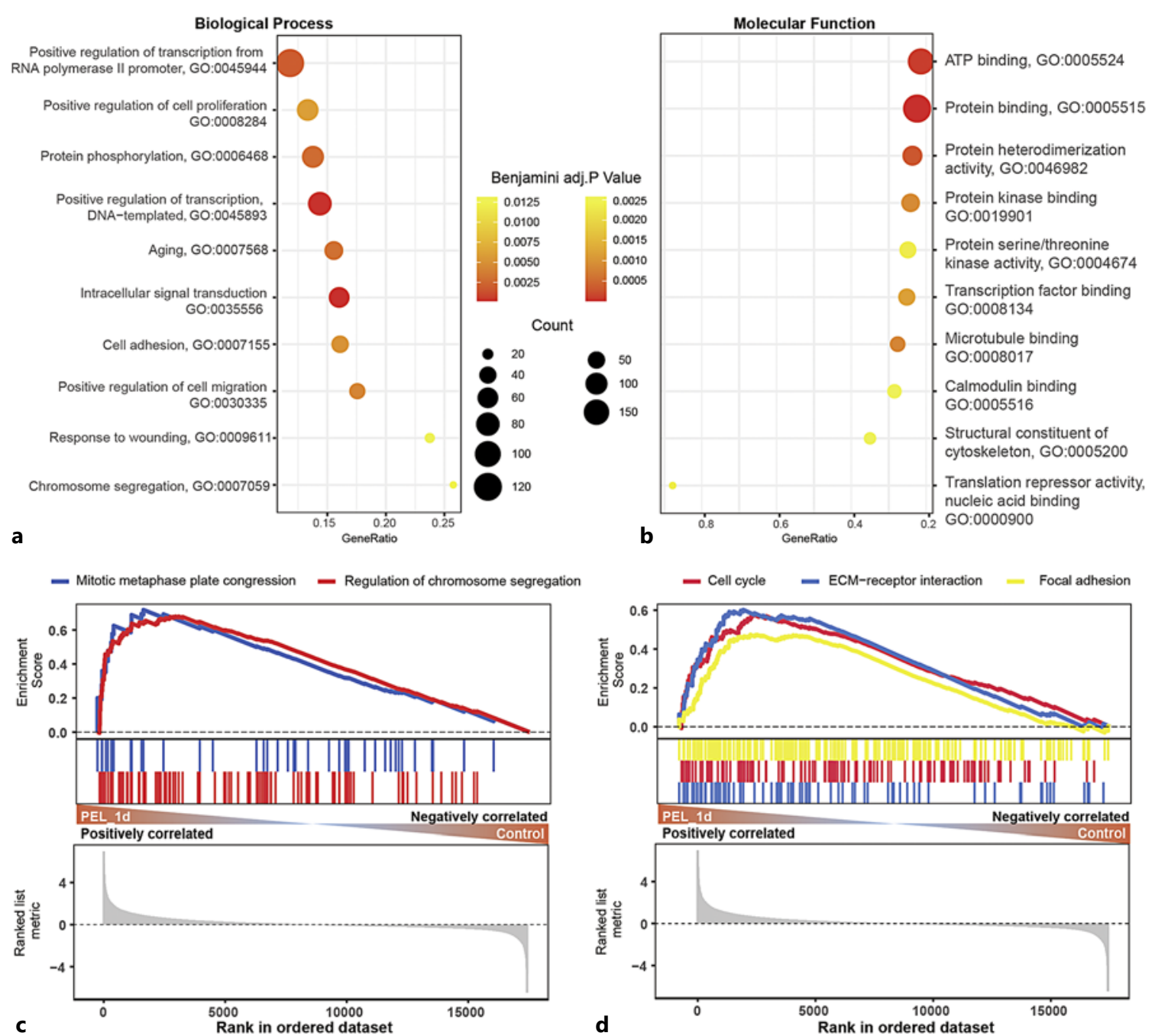

C
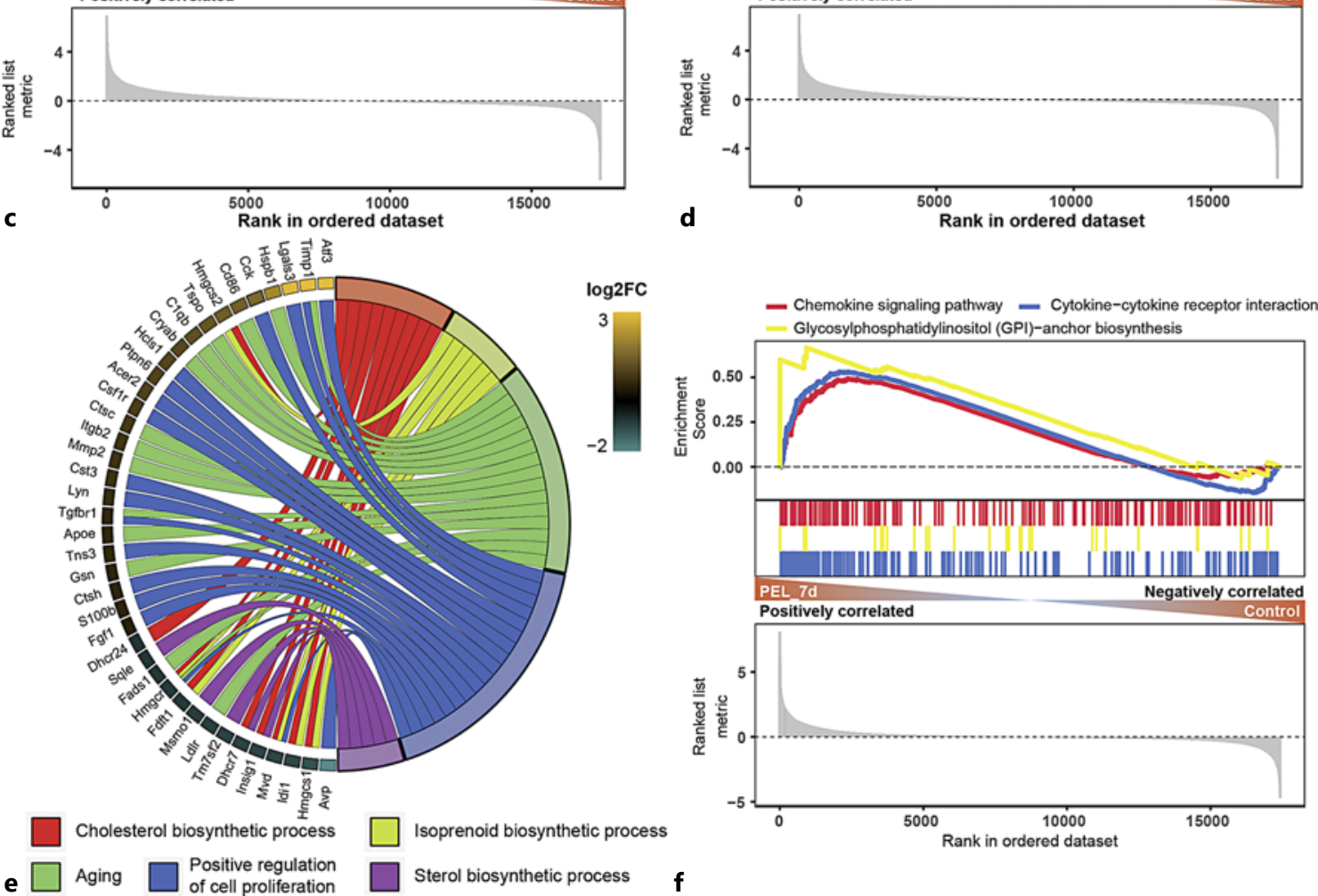

3

(For legend see next page.) 
hypothalamic AVP neurons further validated the association of Atf3/c-Jun/Lgals3 with CDI after pituitary stalk injury (Fig. 4h; online suppl. Fig. S2). Elevated expression of Atf3 and p-c-Jun were also observed in rat hypothalamus after PEL surgery (online suppl. Fig. S3).

Additionally, Lgals3 was found to be upregulated in the mouse hypothalamic AVP neuronal cytoplasm and pericellular matrix after PEL surgery but was nearly absent in the control group (Fig. 5a). We also detected highly elevated Lgals3 protein level and moderate upregulation of Atf3, c-Jun, and p-c-Jun in the rat hypothalamic SON and PVN tissues on days 3, 7, and 14 after PEL surgery, further demonstrating the activation of the Atf3/cJun/Lgals 3 axis $(H=9.574,3$ df; $p<0.05$; Fig. $5 b-c)$. The Lgals3 expression pattern was reminiscent of an autocrine manner similar to cytokines and chemokines and indicates that Lgals3 might function as a secreted extracellular matrix.

\section{Activated Microglia Phagocytize Hypothalamic AVP Neurons}

Lgals3 has previously been shown to activate microglial proliferation and phenotype transition when secreted into the extracellular environment in various conditions [34-36]. To investigate whether Lgals3 could induce microglial activation in our CDI model, we performed staining for the microglia-specific marker Ibal. As shown in Figure $5 \mathrm{~d}-\mathrm{f}$, the number of $\mathrm{Iba}^{+}{ }^{+}$microglia increased rapidly in mouse hypothalamic SON and PVN after PEL surgery (SON: $F_{|4,25|}=54.637$; $p<0.01$; Fig. 5e; PVN: $F_{|4,25|}$ $=57.412 ; p<0.01$; Fig. 5f). These findings were in accor-

Fig. 3. Enrichment analysis identified the involvement of transcription factors in hypothalamic injury. Enriched GO terms of DEGs, including biological processes (a) and molecular function (b), were visualized by bubble plot. GSEA analysis results showed the upregulation of cell cycle and proliferation in PEL_1d group compared with the control group $(\mathbf{c}, \mathbf{d})$. Top 5 enriched biological

Fig. 4. RNA-seq analysis identified the involvement of Atf3/c-Jun/ Lgals3 axis. Venn diagram identified the Atf3 as the only differentially expressed transcription factor at different times after PEL surgery (a). Upregulation of Atf3 in AVP neurons characterized by $\mathrm{Atf}^{+} \mathrm{AVP}^{+}$neurons in mouse hypothalamic SON and PVN after PEL surgery (b), scale bars, $50 \mu \mathrm{m}$ for low magnification images and $20 \mu \mathrm{m}$ for high magnification images. Quantification results of Atf3 positive AVP neurons in SON (c) and PVN (d) $(n=$ 6). Venn diagram identified 9 DEGs to be Atf3 potential target genes (e). PPI network constructed from 9 Atf3 potential target genes and b-ZIP family transcription factors (f). Core module extracted from PPI network in F indicated the Atf3/c-Jun/Lgals3 reg-

Transcriptomic Analysis of Central

Diabetes Insipidus dance with the enrichment of the proliferation-related GO terms and GSEA analysis results as shown in Figure 3. Moreover, $\mathrm{Iba} 1^{+}$microglia exhibited typical amoeboidlike morphology with a high level of branch staining adjacent to AVP neuronal bodies and axonal terminals on days 3 and 7 after PEL surgery. In contrast, almost no microglia was in contact with AVP neurons in the control group. Additionally, elevated expression of another microglial activation marker gene, Spp1 based on RNA-seq data from rat model further validated the microglial activation process in CDI ( $H=7.200,2 \mathrm{df} ; p<0.05$; Fig. $5 \mathrm{~g})$. The mRNA level for several microglial activation marker genes, including TNF- $\alpha$, iNOS, Arg1, and $I L-1 \beta$, in the mouse hypothalamic PVN were also increased after PEL surgery ( $p<0.01$, Fig. $5 \mathrm{~h}-\mathrm{k}$ ). Focusing on the interaction between AVP neurons and activated microglia, we performed $3 \mathrm{D}$ reconstruction using Imaris software and found that AVP neurons were embedded in activated microglia on day 3 after PEL surgery (Fig. 6a). Additionally, we performed 3D optical clearing immunolabeling using the iDISCO method and observed high spatial accumulation of $\mathrm{Iba}^{+}$microglia in the hypothalamic SON and PVN on day 3 after PEL surgery, compared with uniform distribution in the control group (Fig. 6b; online suppl. movies 1-4). Taken together, these findings indicate that microglia might be activated via Atf3/c-Jun/Lgals3-mediated manner after PEL surgery and phagocytize magnocellular AVP neurons, consequently resulting in AVP neuronal clearance and axonal elimination in CDI.

To validate the involvement of Atf3/c-Jun/Lgals3 in microglial activation in the hypothalamic pituitary stalk

processes of DEGs from the PEL_7d group compared with the control group (e). GESA revealed chemokine and cytokine associated pathways enriched in the PEL_7d group (f). PEL, pituitary stalk electric lesion; GO, gene ontology; GSEA, gene set enrichment analysis.

ulatory axis $(\mathbf{g})$. Co-localization staining validated the expression Atf3 and p-c-Jun (activated form of c-Jun encoded by Jun) in mouse SON and PVN after PEL surgery (h), scale bars, $20 \mu \mathrm{m}$ for low magnification images and $10 \mu \mathrm{m}$ for high magnification images. Data are analyzed by 1-way ANOVA followed by LSD multiple comparisons test (c) and Kruskal-Wallis test followed by Dunn's multiple comparisons (d). Data are presented as mean \pm SEM. ${ }^{* *} p<0.01$ compared with control group. PEL, pituitary stalk electric lesion; AVP, arginine vasopressin; SON, supraoptic nucleus; PVN, paraventricular nucleus; PPI, protein-protein interaction; Atf3, activating transcription factor 3; DAPI, diamidino2-phenylindole.

(For figure see next page.)

Neuroendocrinology 2022;112:874-893 883 


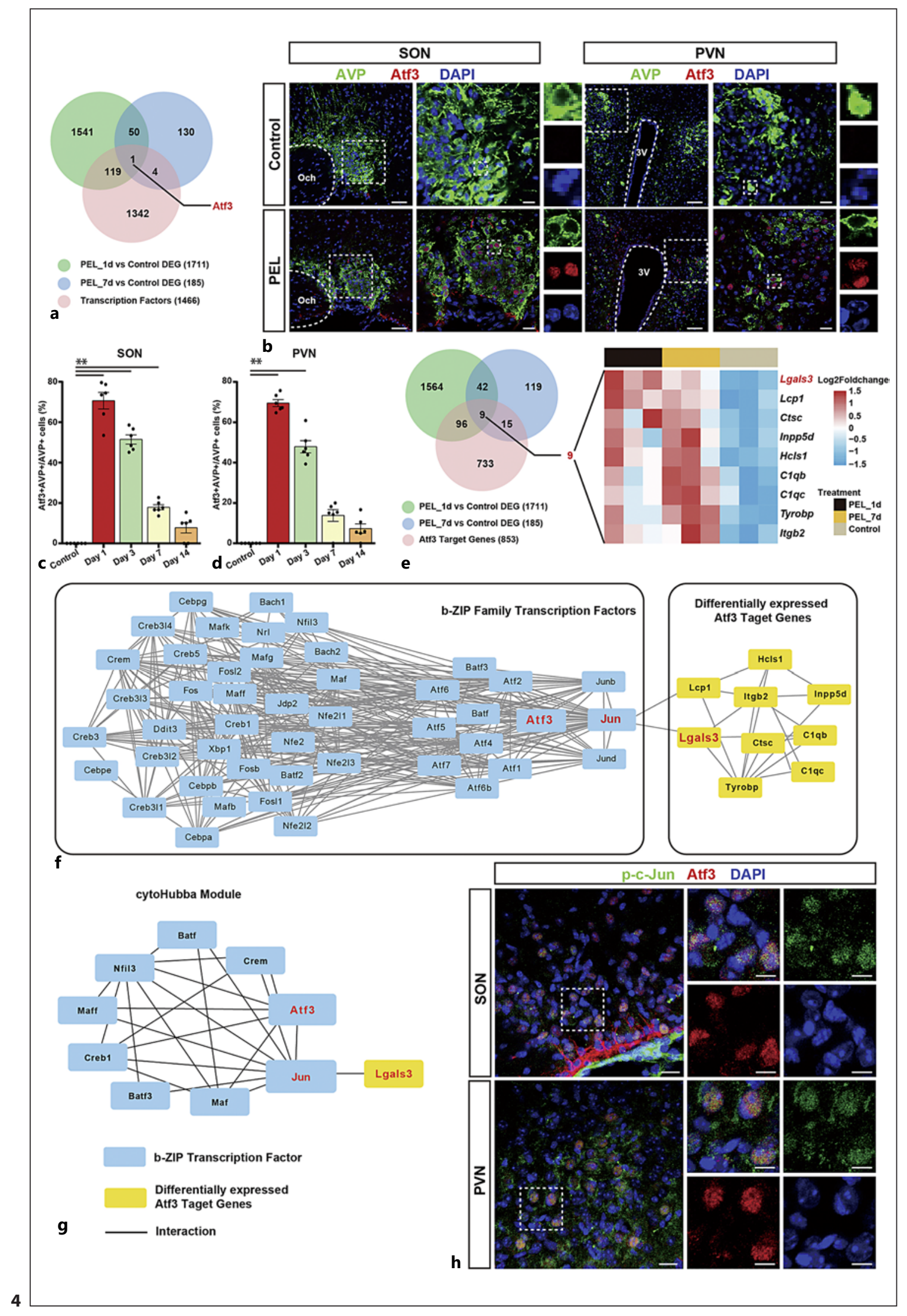



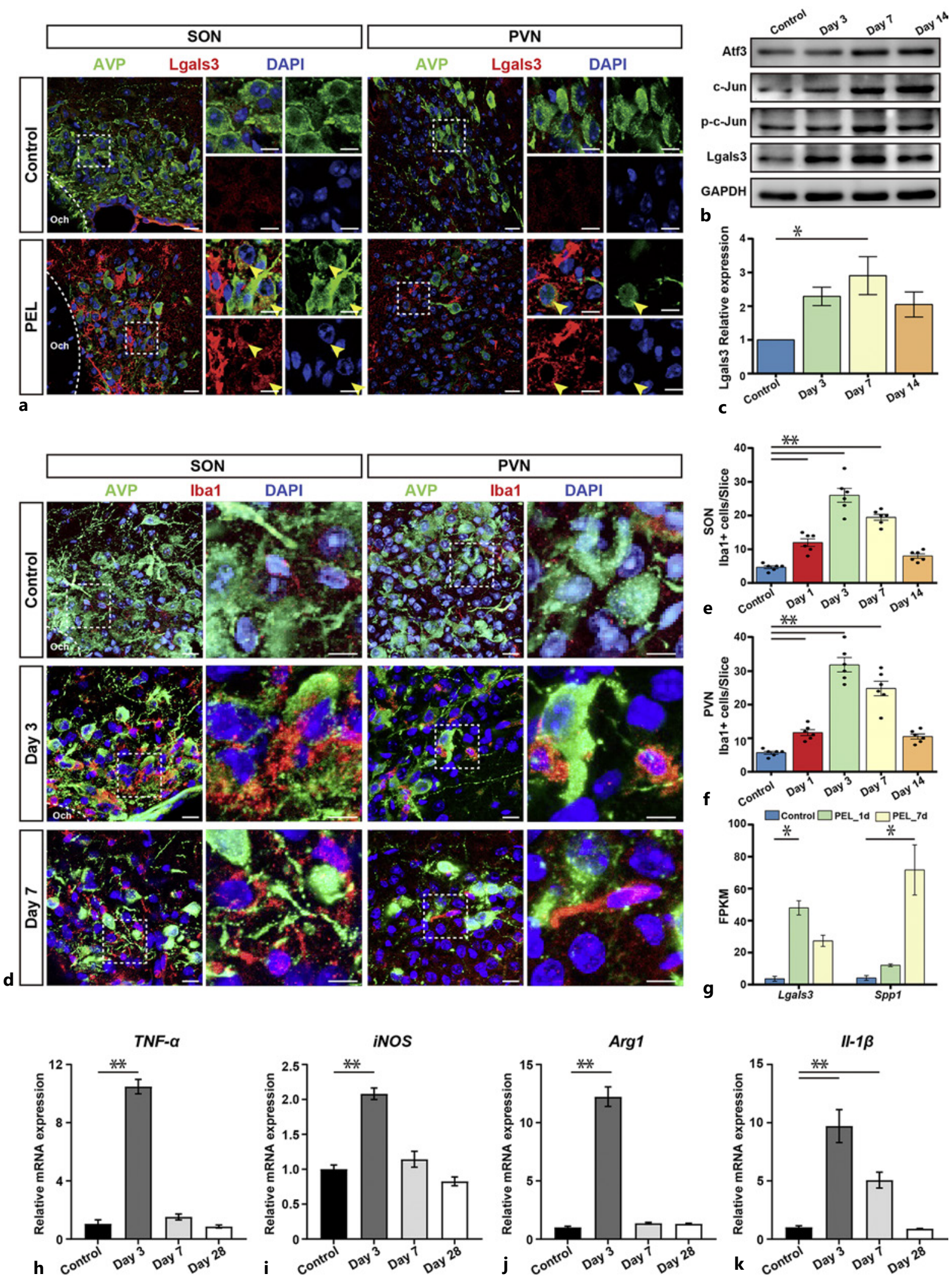

(For legend see next page.) 
injury-induced CDI condition, we intracerebroventricularly (i. c. v.) injected a specific inhibitor of the Atf3/c-Jun pathway (SP600125) to PEL-treated mice via cannula (online suppl. Fig. S4). As shown in Figure 7a-e, inhibition of Atf3/c-Jun quenched the expression of $\mathrm{p}$-c-Jun and Lgals3 in AVP neurons, with decreases in the co-expression ratios (p-c-Jun ${ }^{+} \mathrm{AVP}^{+} / \mathrm{AVP}^{+}$) to $33.6 \%$ in the hypothalamic SON and $28.9 \%$ in the PVN. Furthermore, the number of microglia significantly decreased from 36.0/slicein vehicle-treated mice to 13.7/slice in SP600125treated mice on day 3 after PEL surgery. Furthermore, quantification of residual AVP neurons demonstrated partial recovery in the long-term phase after SP600125 treatment on days 7 and 14 after PEL surgery (Fig. 7f-h). These results indicate that the inhibition of Atf3/c-Jun/ Lgals3 could alleviate microglial activation and hypothalamic AVP neuronal loss after PEL surgery.

In brief, based on transcriptomic RNA-sequencing and bioinformatics analysis, our study demonstrated the upregulation of transcription factor Atf3/c-Jun in CDI after hypothalamic-pituitary stalk injury. Further experiments revealed that Atf3/c-Jun could modulate Lgals3 expression, promote microglial activation, and participate in the process of magnocellular AVP neuronal loss through phagocytosis.

\section{Discussion}

\section{Typical CDIs after Hypothalamic Injury}

Hypothalamic injury caused by trauma, tumor, or surgery has been reported to be associated with various disorders, such as high fever, disturbance of consciousness, and water-electrolyte imbalance $[1,37,38]$. Among these, $\mathrm{CDI}$ is the most common symptom, especially when the pituitary stalk is implicated during tumor resection or other operations near the hypothalamus. As the pituitary stalk serves as the neuron-axon transport path of several neuroendocrine hormones from the hypothalamic mag-

Fig. 5. Microglial activation after Atf3/c-Jun/Lgals3 upregulation. Elevated expression of Lgals3 in mouse hypothalamic AVP neurons cytoplasm, and pericellular location after PEL surgery (a). Immunoblotting validated time-course elevated expression of Atf3, p-c-Jun and Lgals3 in rat hypothalamic tissue after PEL surgery $(\mathbf{b}, \mathbf{c})$. Iba1 staining showed the microglial activation in mouse hypothalamic SON and PVN on days 3 and 7 after PEL surgery (d). Quantification results of Ibal positive microglia in SON (e) and PVN (f) $(n=6)$. RNA-seq data revealed high expression of several marker genes for microglial activation including Spp1 and Lgals3 $(\mathbf{g})(n=3)$. Relative mRNA expression of several microglial activa- nocellular neurons in SON and PVN to the neurohypophysis, pituitary stalk injury could lead to a lack of AVP peptides in peripheral circulation, which results in abnormality of the reabsorption and filtration of water and electrolytes from the kidney and finally causes body fluid homeostasis disorder. Besides antidiuretic peptides, hypothalamic magnocellular neurons could also secrete diuretic peptides, such as OXT, to exert a reciprocal regulation of hydromineral homeostasis. Similar to AVP, OXT is mainly synthesized by magnocellular neurons in the SON and PVN. A recent study also demonstrated that a portion of parvocellular OXT-producing neurons in PVN could project to the ventral tegmental area and substantia nigra, affect the resident dopamine neurons, and thereby participate in the modulation of social behavior [39]. Moreover, other related hormones, atrial natriuretic peptide and B-type natriuretic peptide also participate in the orchestration of water intake and output through the regulation of AVP/OXT balance, and directly modify the neural circuit of the osmoreceptor organ in the brain which sends efferent fibers to magnocellular neurons in SON and PVN [3]. However, due to the lack of proper animal models, few studies have investigated the change of AVP/OXT neurons in hydromineral imbalance issues especially in CDI after hypothalamic injury [19]. Therefore, in this study, we aimed to elucidate the comprehensive change of hypothalamus in the mouse CDI model induced by PEL surgery. Our characterization of the mouse CDI model reveals it to be equivalent to our already published rat model [25], including the similar patterns of water-electrolyte metabolism-associated parameters, such as DWC, DUV, and USG. Moreover, the location of injury site, MRI images, serum AVP contents, and loss of AVP neurons are also validated in mouse model as those in the rat model. Thus, in this study, we performed transcriptomic analysis in rat CDI model in consideration of accurate acquisition and adequate RNA content extraction for sequencing, and subsequently investigated specific genes expression pattern and related pathophysi-

tion related marker genes in mouse hypothalamic PVN after PEL surgery, including TNF- $\alpha(\mathbf{h}), i N O S(\mathbf{i}), \operatorname{Arg} 1(\mathbf{j}), I L-1 \beta(\mathbf{k})(n=3)$. Data are analyzed by 1-way ANOVA followed by LSD multiple comparisons test $(\mathbf{e}, \mathbf{f}, \mathbf{h}-\mathbf{k})$ and Kruskal-Wallis test followed by Dunn's multiple comparisons $(\mathbf{c}, \mathbf{g})$. Scale bars, $20 \mu \mathrm{m}$ for low magnification images and $10 \mu \mathrm{m}$ for high magnification images. Data are presented as mean \pm SEM. ${ }^{*} p<0.05,{ }^{* *} p<0.01$ compared with the control group. PEL, pituitary stalk electric lesion; AVP, arginine vasopressin; SON, supraoptic nucleus; PVN, paraventricular nucleus; Atf3, activating transcription factor 3; DAPI, diamidino2-phenylindole. 
ological changes in mouse CDI model to clarify the underlying regulatory mechanism.

In clinical practice, CDI often occurs when the hypothalamus or sellar region is involved, almost in $17-27 \%$ of craniopharyngioma patients before diagnosis and nearly all postoperative patients when the pituitary stalk is sacrificed during tumor resection $[12,40]$. In this study, we constructed a hypothalamic injury model induced by complete lesion of the pituitary stalk, which was also suitable for studying CDI and injury-induced neuronal loss of the hypothalamus, because the pituitary stalk receives axonal output from magnocellular neurons residing in the hypothalamic SON and PVN. PEL-treated mice exhibited distinguished diabetes insipidus symptoms with high DWC, DUV, and low USG, which were consistent with the typical clinical symptoms of patients suffering from CDI. Furthermore, unlike other complicated symptoms, the PEL model could also serve as an effective tool to evaluate the recovery in the long term based on convenient daily water-electrolyte metabolism-related parameters after hypothalamic injury. Considering the distinct role of AVP/OXT in hydromineral homeostasis regulation, we mainly focused on the AVP neurons due to their antidiuretic function, and found elimination of both magnocellular AVP cell bodies and neuronal fibers in the hypothalamo-neurohypophyseal system and lack of antidiuretic peptides in systemic circulation. This also accords with our transcriptomic observations in rat CDI model, which showed that $A v p$, rather than Oxt, was significantly downregulated. Additionally, these results are consistent with the phenomena that patients suffering from CDI always have low plasma AVP contents, and desmopressin treatment can relieve the polydipsia and polyuria symptoms [41]. Except for magnocellular AVP neurons, it should be taken into account that PVN contains parvocellular neurons in different sub-nuclei dedicated to regulating hydromineral homeostasis, including compensatory activation of the renin-angiotensin-aldosterone system [42], stimulation of the hypothalamic-pituitary-adrenal axis through corticotropin releasing hormone neurons [43], and modulation of central sodium and osmosensors [44]. These could promote the reestablishment of water-electrolyte balance in severe pathological states, such as CDI. However, parvocellular neurons in PVN kept unaffected in our hypothalamic-pituitary stalk lesion model, as they mainly project to the median eminence rather than neurohypophysis, where they secret hypophysiotropic factors into the hypophysial portal circulation.

Transcriptomic Analysis of Central

Diabetes Insipidus
Association of Transcription Factors Atf3/c-Jun with CDI Based on RNA-Seq Analysis

Considering the vital role of hypothalamic SON and PVN in body fluid homeostasis regulation, we used highthroughput RNA sequencing to study the transcriptomic changes in these nuclei using rat CDI model. Our transcriptome data revealed 1,711 DEGs (1,292 upregulated and 419 downregulated) on day 1 and 185 DEGs (150 upregulated and 35 downregulated) on day 7 after PEL surgery. Among these DEGs lists, we found 610 DEGs were associated with salt loading-induced hyperosmolality and 312 DEGs were in common with water deprivation-induced hyperosmolality, whereas only 20 DEGs were reported to be responsive to sodium depletion-induced hypoosmolality based on previous studies $[19,20]$. These global gene expression patterns also support the phenomenon that patients suffering from CDI always have slightly elevated plasma osmolality in clinical practice [45]. To reestablish body fluid homeostasis in CDI, the transcriptomic remodeling of the SON and PVN might modulate the neuropeptides AVP and OXT production and secretion. However, in contrast to the upregulation of $A v p$ under hyperosmolality, our transcriptomic data demonstrated the drastically decreased expression of Avp. This inconsistency may be explained by the fact that although $A v p$ transcription and expression of single magnocellular neuron might be activated, substantial loss of cells could lead to the gloal downregulation of $A v p$, in accordance with our immunostaining results showing the loss of AVP positive magnocellular neuronal bodies, axonal fibers in the SON, PVN, and neurohypophysis in mouse CDI model. Moreover, it should be taken into account that due to the limitation of the bulk tissue RNA sequencing, we could only evaluate the average expression pattern in hypothalamic SON and PVN, rather than distinguishing the specific gene expression difference between magnocellular and parvocellular neurons, including melanin concentrating hormone, thyrotropin releasing hormone neurons in PVN. So, we further

Fig. 6. Activated microglia phagocytized AVP neurons after hypothalamic injury. 3D simulation of $\mathrm{Iba}^{+}$microglia and AVP neurons using "surface" method of Imaris software in mouse hypothalamic SON and PVN on day 3 after PEL surgery (a), respectively, scale bars, $20 \mu \mathrm{m}$ for low magnification images and $10 \mu \mathrm{m}$ for high magnification images. The spatial accumulation of microglia in mouse hypothalamic SON and PVN using immunolabeling-enabled 3D imaging of solvent-cleared organs (iDISCO) method (b), scale bar, $200 \mu \mathrm{m}$. SON, supraoptic nucleus, PVN, paraventricular nucleus; PEL, pituitary stalk electric lesion; AVP, arginine vasopressin; DAPI, diamidino-2-phenylindole.

(For figure see next page.)

Neuroendocrinology 2022;112:874-893 887 

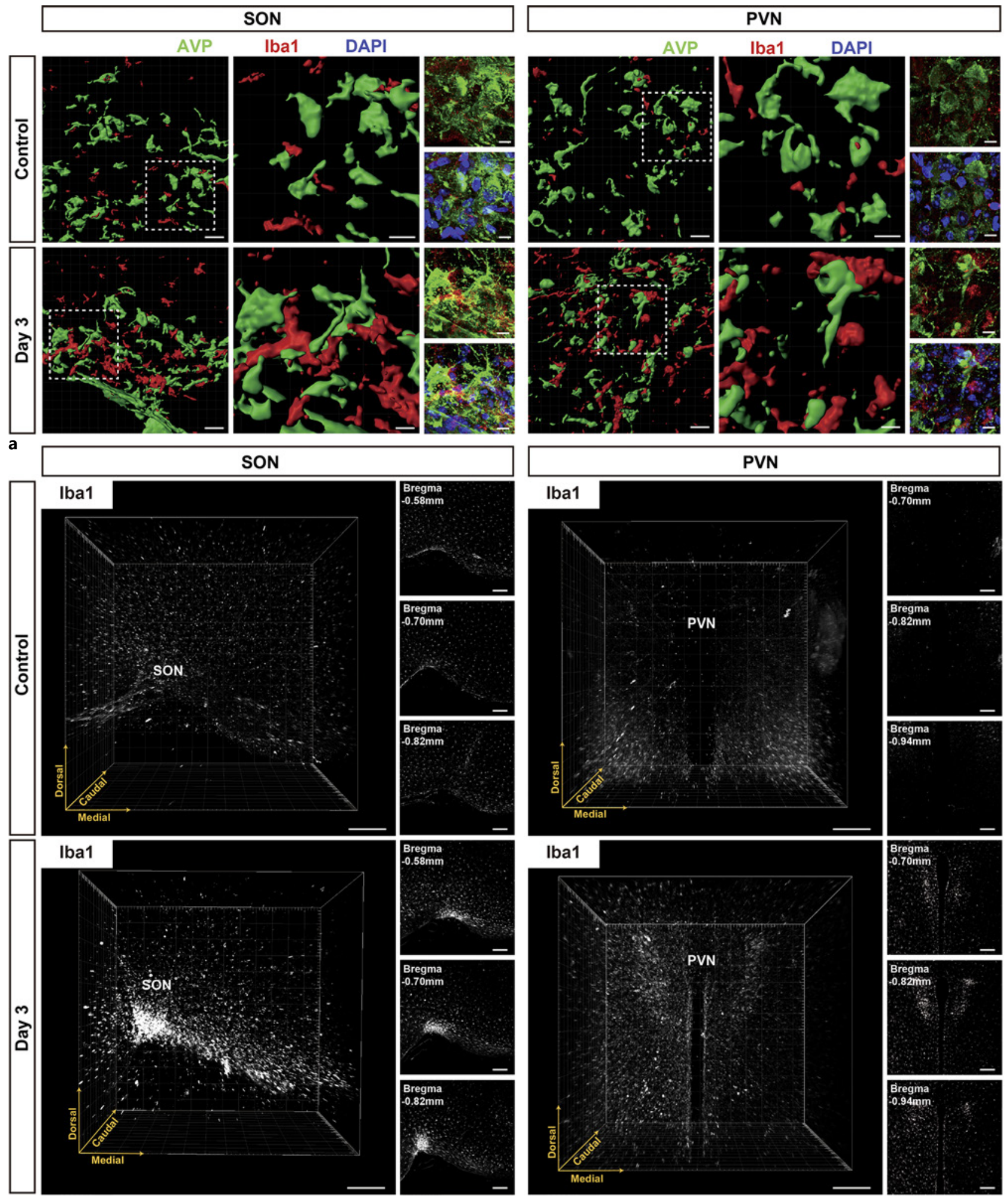

6

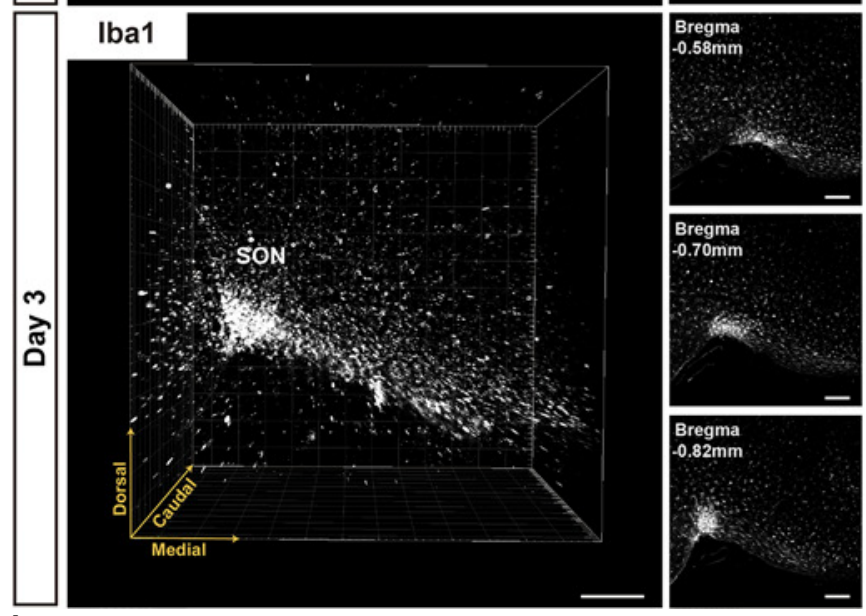

b 

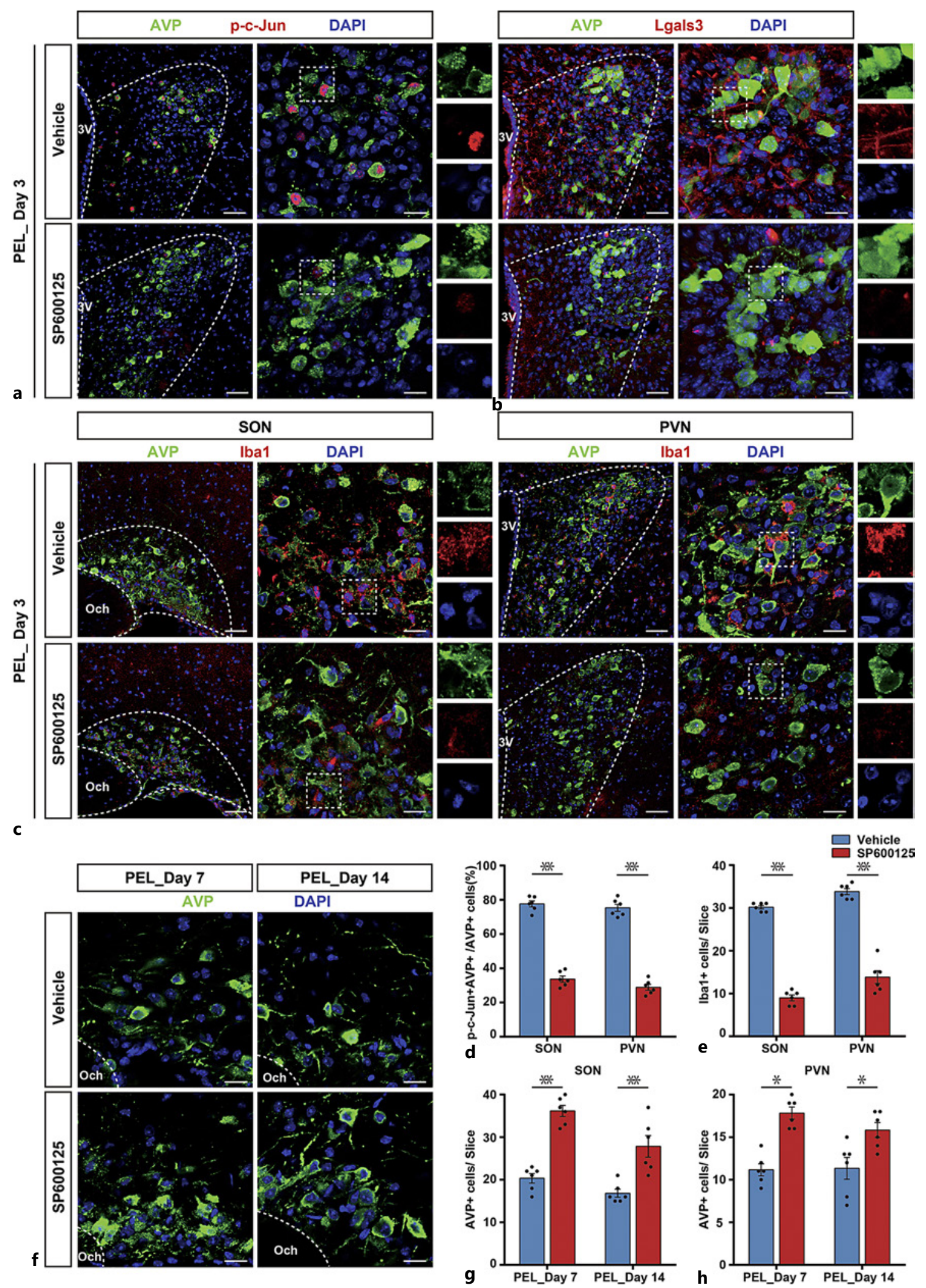

(For legend see next page.) 
validated the expression of several DEGs in magnocellular AVP-producing neurons both in rat and mouse model. Future studies using singel-cell sequencing may provide a new insight into the cellular heterogeneity and specific gene expression profile of single magnocellular and parvocellular neuron in response to the osmotic stimuli and related issues.

Previous studies have discussed the function of transcription factors in the osmotic regulation, including the initiation of acute cellular response and regulation of neuropeptide expression, especially the b-ZIP family members. Among a total of 7,188 DEGs involved in salt loading-induced hyperosmolality, 342 genes were identified as transcription factors with $24 \mathrm{~b}$-ZIP family members [20]. Consistently, our transcriptome data in CDI also revealed 124 transcription factors were differentially expressed, including $13 \mathrm{~b}$-ZIP family members (Atf3, Atf7, Creb1, Creb3l2, Cebpa, Cebpb, Cebpd, Dbp, Bach1, Bach2, Maf, Maff, Mafg). Among these, Creb1 and Creb3l2 belong to the cAMP response element binding protein family, which has been shown previously to regulate the AVP expression in hypothalamic SON and PVN [46]. Thus, it is possible that in hypothalamic-pituitary stalk injury-induced CDI, loss of AVP neurons leads to compensatory elevated expression of several $A v p$-related regulatory transcription factors, such as Creb1 and Creb3l2 in residual magnocellular neurons. As stress-induced genes, Atf 3 and Atf7 are involved in various stimulus-response processes, such as traumatic injury, endoplasmic reticulum stress, and oxidative stress [47]. Recent studies have also identified the upregulation of several endoplasmic reticulum stress-related genes, such as Atf3, Atf4, Atf5, Chac1, and Chop after retinal ganglion cell axonal injury [48]. Consistently, our RNA-seq analysis also revealed upregulation of Atf3 after PEL surgery. Subsequent GO and GSEA analyses further emphasized the vital role of the transcription factor in hypothalamic injuryinduced CDI based on the enrichment of several transcriptional regulation processes, such as regulation of

Fig. 7. Inhibition of Atf3/c-Jun/Lgals3 regulatory axis alleviated the AVP neuronal loss. Inhibition of Atf3/c-Jun by specific inhibitor SP600125 quenched the rapid expression of p-c-Jun in mouse hypothalamic AVP neurons on day 3 after PEL surgery (a). Inhibition of Atf3/c-Jun by specific inhibitor SP600125 quenched the expression of Lgals3 (b). Inhibition of Atf3/c-Jun by specific inhibitor SP600125 relieved the microglial activation in mouse hypothalamus after PEL surgery $(\mathbf{c})(n=6)$, scale bars, $50 \mu \mathrm{m}$ for low magnification images and $20 \mu \mathrm{m}$ for high magnification images. Quantification results of $\mathrm{p}$-c-Jun positive AVP neurons of a (d) transcription from RNA polymerase II promoter (GO: 0045944) and positive regulation of transcription DNAtemplate (GO: 0045893). Furthermore, Atf3 was identified as the only differentially expressed transcription factor both in PEL_day 1 and PEL_day 7 groups, based on an established list consisting of rat and human transcription factors [49]. Consistent with previously mentioned in hyperosmotic issues, we observed that there were 77 common differentially expressed TFs in rat TF list, human TF list and DEGs from our CDI model. Among these 77 TFs, we found the 12 b-ZIP family TFs (Atf3, Atf7, Creb1, Cebpa, Cebpb, Cebpd, Dbp, Maf, Maff, Mafg, Bach1, Bach2) comprised the largest portion, which also indicates the important role of b-ZIP family TFs in AVP expression and hydromineral homeostasis regulation in CDI.

Atf3, an immediate early transcription factor gene belonging to the activating protein 1 (AP-1) family, is classified as a basic leucine zipper (b-ZIP) transcription factor and functions by binding to the canonical ATF/CRE site $\left(5^{\prime}\right.$-TGACGTCA- $\left.3^{\prime}\right)$ or a similar AP-1 site $\left(5^{\prime}\right.$-TGA(C/G) TCA-3') $[47,50]$. As a typical stress-induced transcription factor, Atf3 is triggered by a series of external signals, such as axonal injury, DNA damage, chemotherapeutic drugs, and ischemia-reperfusion. Upon neural injury, elevated expression of Atf3 during a short period of time could regulate the expression of target genes through binding other transcription factors such as c-Jun and Atf2, and participate in multiple biological processes such as injury response, neuronal loss, axonal elimination, and tissue reconstruction $[47,50]$. Co-expression of another b-ZIP family transcription factor c-Jun with Atf3, based on PPI network core module analysis and costaining experiment also validated the expression of Atf3 and associated regulatory network. Indeed, the Atf3/cJun axis has also been reported to be involved in several other neural injury associated issues [15]. However, few studies have clarified the downstream target genes and their related pathophysiological process.

$(n=6)$. Quantification results of Ibal positive microglia of $\mathbf{c}(\mathbf{e})(n$ =6). Residual functional AVP neurons in the longterm after inhibition of Atf3/c-Jun by specific inhibitor SP600125 (f), scale bar, $20 \mu \mathrm{m}$. Quantification results of AVP neurons in hypothalamic SON $(\mathbf{g})$ and PVN $(\mathbf{h})(n=6)$. Data are analyzed by unpaired $t$ test $(\mathbf{d}, \mathbf{e}, \mathbf{g}, \mathbf{h})$. Data are presented as mean \pm SEM. ${ }^{*} p<0.05,{ }^{* *} p<$ 0.01 compared with the vehicle group. PEL, pituitary stalk electric lesion; SON, supraoptic nucleus; PVN, paraventricular nucleus; AVP, arginine vasopressin; Atf3, activating transcription factor 3; DAPI, diamidino-2-phenylindole. 
Microglial Activation Induced by the Atf3/c-Jun/

Lgals3 Axis

Except for direct regulation of magnocellular neurons, glial cells have been reported to be involved in body fluid homeostasis maintenance through modulating neuropeptides secretion from neurons and neurohypophysis. These included the effects of astrocytes on neurotransmitter release and uptake, and the microglial function on synaptic pruning. In fact, based on our transcriptomic data and previous studies, we observed the upregulation of several glial activation and inflammation-related genes, including Gfap, S100b, C3, Spp1, in the hydromineral imbalance [19-22]. In addition, recent studies have also revealed the involvement of several transcription factors in injury-induced biological processes, such as microglial phenotype transition, neuronal clearance, debris removal, and gliosis [51-53]. In this study, using the Cistrome database and core hub module analysis, Lgals3 was identified as the most significant target gene of Atf3/c-Jun, and relatively elevated expression of Lgals3 was found in hypothalamic SON and PVN after PEL surgery. Upon CNS assault, Lgals3 regulates several biological processes, including neuronal apoptosis and inflammation, and activates microglia, especially in trauma and axonal injury [54]. Involvement of Lgals3 in microglial morphology transition from branched to amoeboid was reported in traumatic brain injury and optic nerve injury through K-Ras signaling pathways, neuroinflammation response, or induction of apoptosis [55]. In addition, several other potential target genes of Atf3, including Ctsc, C1qb, Clqc, and Tyrobp revealed by our RNA-seq data from rat CDI model, were also reported to participate in the microglial activation process through promoting the proliferation, morphological change, and the phagocytic ability [56]. In the present study, we found that Lgals3 overexpression was indeed correlated with microglial activation and could be reversed by treatment with Atf3/c-Jun inhibitor in mouse CDI model, further confirming the role of the Atf3/c-Jun/ Lgals3 axis in the microglial activation process after hypothalamic injury. Moreover, coinciding with microglial phagocytosis in CNS disorders, such as trauma, neurodegenerative disease, and brain tumors [57, 58], our findings showed that activated microglia exhibited typical amoeboid morphology and maintained close contact with the AVP neuronal bodies and axonal terminals, indicating the clearance of neurons was occurring. In addition, considering the wide distribution of microglia in the CNS, we demonstrated the spatial accumulation of microglia in the SON and PVN after hypothalamic injury using the tissue clearing method iDISCO [28]. Except for morphological validation, we also observed significant upregulation of microglial activation-

Transcriptomic Analysis of Central

Diabetes Insipidus related genes, including Ctsc, Ttbk2, Trem2, Aif, along with downregulation of microglial inhibition-related genes, including Nr1d1, Ldlr in our transcriptomic data [59-61]. In addition, differential expression of Cstc, Tyrobp, Nr1d1, Lgals3, C1qb, C1qc, and C3 were also validated in hyperosmotic or hypoosmotic conditions [19-22]. Nevertheless, more studies are necessary to investigate the role of these microglia-related genes in magnocellular neuron gene expression, activity and body fluid homeostasis modulation under various osmotic stimuli.

\section{Conclusion}

The present work presents a global transcriptomic profile of CDI induced by hypothalamic-pituitary stalk injury, and investigates the expression patterns of several transcription factors likely to be implicated in the regulation of AVP neuronal changes and related pathophysiological processes in this body fluid imbalance condition. In summary, our findings indicate that Atf3/c-Jun/Lgals3 axis is associated with the microglial activation and hypothalamic AVP neuronal loss in CDI. However, to explore specific potential therapeutic targets, further research is needed to clarify the complex cascade of alterations in molecular, cellular, and regulatory networks in CDI and related issues after hypothalamic injury.

\section{Acknowledgments}

The authors would like to thank Figure Ya for technical assistance in analysis of RNA-seq data and visualization.

\section{Statement of Ethics}

All animal procedures were approved by the Nanfang Hospital ethics committee (No. NFYY-2018-47) and conducted in accordance with Guide for the Care and Use of Laboratory Animals (NIH Publication).

\section{Conflict of Interest Statement}

The authors have no conflicts of interest to declare.

\section{Funding Sources}

This study was supported by Guangdong Basic and Applied Basic Research Foundation (2020A1515110028, 2020A1515110564), National Natural Science Foundation of China (81900709), Science 
and Technology Planning Project of Guangzhou (201902020017), President Foundation of Nanfang Hospital, Southern Medical University (2019C001, 2019C016).

\section{Author Contributions}

Z.F. and S.Q. designed experiments; M.Z., Y.O., G.W., K.L., and J.P. performed experiments; X.W., M.C., D.G., and P.N. analyzed data; M.Z. and G.W. wrote the manuscript; Y.L. and S.Q. revised the manuscript.

\section{References}

1 Alharfi IM, Stewart TC, Foster J, Morrison GC, Fraser DD. Central diabetes insipidus in pediatric severe traumatic brain injury. Pediatr Crit Care Med. 2013;14:203-9.

2 Edate S, Albanese A. Management of electrolyte and fluid disorders after brain surgery for pituitary/suprasellar tumours. Horm Res Paediatr. 2015;83:293-301.

3 Mecawi Ade S, Ruginsk SG, Elias LL, Varanda WA, Antunes-Rodrigues J. Neuroendocrine regulation of hydromineral homeostasis. Compr Physiol. 2015;5:1465-516.

4 Augustine RA, Seymour AJ, Campbell RE, Grattan DR, Brown CH. Integrative neurohumoral regulation of oxytocin neuron activity in pregnancy and lactation. J Neuroendocrinol. 2018.

5 Wang L, Moenter SM. Differential roles of hypothalamic AVPV and arcuate kisspeptin neurons in estradiol feedback regulation of female reproduction. Neuroendocrinology. 2020;110(3-4):172-84.

6 Ai PH, Chen S, Liu XD, Zhu XN, Pan YB, Feng DF, et al. Paroxetine ameliorates prodromal emotional dysfunction and late-onset memory deficit in Alzheimer's disease mice. Transl Neurodegener. 2020;9:18.

7 Chang CY, Liang MZ, Chen L. Current progress of mitochondrial transplantation that promotes neuronal regeneration. Transl Neurodegener. 2019;8:17.

8 Hemberg M. Summing up the parts of the hypothalamus. Nat Neurosci. 2017;20:378-9.

9 Krasne FB. General disruption resulting from electrical stimulus of ventromedial hypothalamus. Science. 1962;138:822-3.

10 Meyer-Lindenberg A, Domes G, Kirsch P, Heinrichs M. Oxytocin and vasopressin in the human brain: social neuropeptides for translational medicine. Nat Rev Neurosci. 2011;12: 524-38.

11 Waterson MJ, Horvath TL. Neuronal regulation of energy homeostasis: beyond the hypothalamus and feeding. Cell Metab. 2015;22: 962-70.

12 Muller HL. The diagnosis and treatment of craniopharyngioma. Neuroendocrinology. 2020;110:753-66

13 Muller HL. Craniopharyngioma. Endocr Rev. 2014;35:513-43.

\section{Data Availability Statement}

Original RNA seq data can be acquired from the pubic GEO database (GSE167904). Other data that support the findings of this study are available from the corresponding author upon reasonable request.
14 Christ-Crain M. Diabetes insipidus: new concepts for diagnosis. Neuroendocrinology. 2020;110(9-10):859-67.

15 Chen J, Zhang L, Liu L, Yang X, Wu F, Gan X, et al. Acupuncture treatment reverses retinal gene expression induced by optic nerve injury via rna sequencing analysis. Front Integr Neurosci. 2019;13:59.

16 Libby R, Yasuda M, Tanaka Y, Ryu M, Tsuda $\mathrm{S}$, Nakazawa T. Rna sequence reveals mouse retinal transcriptome changes early after axonal injury. PLoS One. 2014;9:e93258.

17 Wang W, Su Y, Tang S, Li H, Xie W, Chen J, et al. Identification of noncoding rna expression profiles and regulatory interaction networks following traumatic spinal cord injury by sequence analysis. Aging. 2019;11:235268.

18 Tran AP, Warren PM, Silver J. The biology of regeneration failure and success after spinal cord injury. Physiol Rev. 2018;98(2):881-917.

19 Dutra SGV, Paterson A, Monteiro LRN, Greenwood MP, Greenwood MP, Amaral LS, et al. Physiological and transcriptomic changes in the hypothalamic-neurohypophysial system after $24 \mathrm{~h}$ of furosemide-induced sodium depletion. Neuroendocrinology. 2021; 111:70-86.

20 Greenwood MP, Mecawi AS, Hoe SZ, Mustafa MR, Johnson KR, Al-Mahmoud GA, et al. A comparison of physiological and transcriptome responses to water deprivation and salt loading in the rat supraoptic nucleus. Am J Physiol Regul Integr Comp Physiol. 2015;308: R559-68.

21 Johnson KR, Hindmarch CC, Salinas YD, Shi Y, Greenwood M, Hoe SZ, et al. A rna-seq analysis of the rat supraoptic nucleus transcriptome: effects of salt loading on gene expression. PLoS One. 2015;10(4):e0124523.

22 Mutsuga N, Shahar T, Verbalis JG, Xiang CC, Brownstein MJ, Gainer H. Regulation of gene expression in magnocellular neurons in rat supraoptic nucleus during sustained hypoosmolality. Endocrinology. 2005;146:1254-67.

23 Feng Z, Ou Y, Zhou M, Wu G, Ma L, Bao Y, et al. A rat model for pituitary stalk electric lesion-induced central diabetes insipidus: application of $3 \mathrm{~d}$ printing and further outcome assessments. Exp Anim. 2018;67:383-92.
24 Feng Z, Ou Y, Zhou M, Wu G, Ma L, Zhang $\mathrm{Y}$, et al. Functional ectopic neural lobe increases gap-43 expression via pi3k/akt pathways to alleviate central diabetes insipidus after pituitary stalk lesion in rats. Neurosci Lett. 2018;673:1-6.

25 Zhou MF, Feng ZP, Ou YC, Peng JJ, Li K, Gong HD, et al. Endoplasmic reticulum stress induces apoptosis of arginine vasopressin neurons in central diabetes insipidus via pi3k/ akt pathway. CNS Neurosci Ther. 2019;25: 562-74.

26 Cranley R. Multivariate analysis: methods and applications. J R Stat Soc Ser C. 1985;34: 290-1.

27 Franklin K, Paxinos G. The mouse brain in stereotaxic coordinates, compact. 2001.

28 Renier N, Wu Z, Simon DJ, Yang J, Ariel P, Tessier-Lavigne M. Idisco: a simple, rapid method to immunolabel large tissue samples for volume imaging. Cell. 2014;159:896-910.

29 Ou Y, Zhou M, Wu G, Gong H, Li K, Wang $\mathrm{X}$, et al. A retaining sphenoid and dura procedure in the rat to obtain intact pituitary-infundibulum-hypothalamus preparations. J Neurosci Methods. 2020;338:108694.

30 Yu G, Wang LG, Han Y, He QY. Clusterprofiler: an $\mathrm{r}$ package for comparing biological themes among gene clusters. OMICS. 2012; $16: 284-7$.

31 Jiao X, Sherman BT, Huang da W, Stephens R, Baseler MW, Lane HC, et al. DAVID-WS: a stateful web service to facilitate gene/protein list analysis. Bioinformatics. 2012;28:1805-6.

$32 \mathrm{Hu} \mathrm{H}$, Miao YR, Jia LH, Yu QY, Zhang Q, Guo AY. Animaltfdb 3.0: a comprehensive resource for annotation and prediction of animal transcription factors. Nucleic Acids Res. 2019;47:D33-38.

33 Mei S, Qin Q, Wu Q, Sun H, Zheng R, Zang $\mathrm{C}$, et al. Cistrome data browser: a data portal for chip-seq and chromatin accessibility data in human and mouse. Nucleic Acids Res. 2017;45:D658-662.

34 Burguillos Miguel A, Svensson M, Schulte T, Boza-Serrano A, Garcia-Quintanilla A, Kavanagh $\mathrm{E}$, et al. Microglia-secreted galectin-3 acts as a toll-like receptor 4 ligand and contributes to microglial activation. Cell Rep. 2015;10:1626-38. 
35 Lalancette-Hebert M, Swarup V, Beaulieu JM, Bohacek I, Abdelhamid E, Weng YC, et al. Galectin-3 is required for resident microglia activation and proliferation in response to ischemic injury. J Neurosci. 2012;32:10383-95.

36 Rahimian R, Béland LC, Kriz J. Galectin-3: mediator of microglia responses in injured brain. Drug Discov Today. 2018;23:375-81.

37 Meier K, Lee K. Neurogenic fever. J Intensive Care Med. 2017;32:124-9.

38 Tang H, Zhu Q, Li W, Qin S, Gong Y, Wang $\mathrm{H}$, et al. Neurophysiology and treatment of disorders of consciousness induced by traumatic brain injury: orexin signaling as a potential therapeutic target. Curr Pharm Des. 2019;25:4208-20.

39 Xiao L, Priest MF, Nasenbeny J, Lu T, Kozorovitskiy Y. Biased oxytocinergic modulation of midbrain dopamine systems. Neuron. 2017;95:368-e5.

40 Müller HL, Merchant TE, Warmuth-Metz M, Martinez-Barbera JP, Puget S, Craniopharyngioma. Nat Rev Dis Primers. 2019;5:75.

41 Garrahy A, Moran C, Thompson CJ. Diagnosis and management of central diabetes insipidus in adults. Clin Endocrinol. 2019;90:2330.

42 Resch JM, Fenselau H, Madara JC, Wu C, Campbell JN, Lyubetskaya A, et al. Aldosterone-sensing neurons in the nts exhibit statedependent pacemaker activity and drive sodium appetite via synergy with angiotensin ii signaling. Neuron. 2017;96:190-206.

43 Carlson DE, Dornhorst A, Seif SM, Robinson AG, Gann DS. Vasopressin-dependent and -independent control of the release of adrenocorticotropin. Endocrinology. 1982;110:680-

44 Porcari CY, Debarba LK, Amigone JL, Caeiro $\mathrm{XE}$, Reis LC, Cunha TM, et al. Brain osmosodium sensitive channels and the onset of sodium appetite. Horm Behav. 2020;118: 104658.
45 Fenske W, Refardt J, Chifu I, Schnyder I, Winzeler B, Drummond J, et al. A copeptinbased approach in the diagnosis of diabetes insipidus. N Engl J Med. 2018;379:428-39.

46 Greenwood M, Bordieri L, Greenwood MP, Rosso Melo M, Colombari DS, Colombari E, et al. Transcription factor creb3l1 regulates vasopressin gene expression in the rat hypothalamus. J Neurosci. 2014;34:3810-20.

47 Rohini M, Haritha Menon A, Selvamurugan $\mathrm{N}$. Role of activating transcription factor 3 and its interacting proteins under physiologi$\mathrm{cal}$ and pathological conditions. Int J Biol Macromol. 2018;120:310-7.

48 Yasuda M, Tanaka Y, Ryu M, Tsuda S, Nakazawa T. RNA sequence reveals mouse retinal transcriptome changes early after axonal injury. PLoS One. 2014;9:e93258.

49 Lambert SA, Jolma A, Campitelli LF, Das PK, Yin Y, Albu M, et al. The human transcription factors. Cell. 2018;175:598-9.

50 Hai T, Wolford CC, Chang YS. Atf3, a hub of the cellular adaptive-response network, in the pathogenesis of diseases: is modulation of inflammation a unifying component? Gene Expr. 2010;15:1-11.

51 Norris GT, Smirnov I, Filiano AJ, Shadowen HM, Cody KR, Thompson JA, et al. Neuronal integrity and complement control synaptic material clearance by microglia after cns injury. J Exp Med. 2018;215:1789-801.

$52 \mathrm{Cao} \mathrm{L}, \mathrm{He}$ C. Polarization of macrophages and microglia in inflammatory demyelination. Neurosci Bull. 2013;29:189-98.

53 Qin C, Zhou LQ, Ma XT, Hu ZW, Yang S, Chen $M$, et al. Dual functions of microglia in ischemic stroke. Neurosci Bull. 2019;35:92133.
54 Blanco Martinez A, da Costa Calaza K, Mendonca H, Carpi-Santos R. Neuroinflammation and oxidative stress act in concert to promote neurodegeneration in the diabetic retina and optic nerve: galectin-3 participation. Neural Regen Res. 2020;15:625.

55 Reichert F, Rotshenker S. Galectin-3 (mac-2) controls microglia phenotype whether amoeboid and phagocytic or branched and nonphagocytic by regulating the cytoskeleton. Front Cell Neurosci. 2019;13:90.

56 Li J, Zhao R, Jiang Y, Xu Y, Zhao H, Lyu X, et al. Bilberry anthocyanins improve neuroinflammation and cognitive dysfunction in app/ psen 1 mice via the cd33/trem2/tyrobp signaling pathway in microglia. Food Funct. 2020; 11:1572-84.

57 Cai Q, Li Y, Pei G. Polysaccharides from ganoderma lucidum attenuate microglia-mediated neuroinflammation and modulate microglial phagocytosis and behavioural response. J Neuroinflammation. 2017;14:63.

$58 \mathrm{Fu}$ R, Shen Q, Xu P, Luo JJ, Tang Y. Phagocytosis of microglia in the central nervous system diseases. Mol Neurobiol. 2014;49:142234.

59 Liu Q, Zhang Y, Liu S, Liu Y, Yang X, Liu G, et al. Cathepsin c promotes microglia $\mathrm{m} 1$ polarization and aggravates neuroinflammation via activation of $\mathrm{ca}(2+)$-dependent $\mathrm{pkc} /$ p38mapk/nf-kb pathway. J Neuroinflammation. 2019;16:10.

60 Ikezu S, Ikezu T. Tau-tubulin kinase. Front Mol Neurosci. 2014;7:33.

61 Griffin P, Sheehan PW, Dimitry JM, Guo C, Kanan MF, Lee J, et al. Rev-erba mediates complement expression and diurnal regulation of microglial synaptic phagocytosis. Elife. 2020;9:e58765. 